\title{
Characterizing robust solutions to monotone games
}

By

\author{
Anne-Christine Barthel \\ West Texas A\&M University \\ Canyon, TX, 79016, USA \\ abarthel@wtamu.edu
}

\author{
Eric Hoffmann \\ West Texas A\&M University \\ Canyon, TX, 79016, USA \\ ehoffmann@wtamu.edu
}

\author{
Tarun Sabarwal \\ University of Kansas \\ Lawrence, KS 66045, USA \\ sabarwal@ku.edu
}

\begin{abstract}
In game theory, $p$-dominance and $p$-best response sets serve as important robustness solution concepts by allowing for deviations from the stringent common knowledge requirements of Nash equilibrium. However, solving for such sets remains largely intractable beyond the simplest of settings. The contributions of this paper are twofold: First, in monotone games, (which include the broad class of supermodular games, submodular games, and their combinations,) we show that these concepts can be characterized in terms of pure strategy Nash equilibria in an auxiliary game of complete information. This makes it considerably easier to compute such sets, facilitating their broader use. Second, these characterizations lead to new results about the structure of entire classes of such solution concepts, including minimal $p$-best response ( $p$-MBR) sets, which generalize wellknown results for pure strategy Nash equilibria. In games with strategic complements, these classes are complete lattices. More generally, they are totally unordered. Several examples highlight the results.
\end{abstract}

JEL Numbers: C60, C70, C72

Keywords: strategic complements, strategic substitutes, monotone games, p-dominance, p-best response set

First Draft: October 2018

This Version: July 22, 2020 


\section{Introduction}

A long-standing problem in game theory is robustness of Nash equilibrium to deviations from complete information and common knowledge. Epistemic conditions for Nash equilibrium play require that players hold correct beliefs (of all orders) about what their opponents will play and are certain about all payoffs. If these are not satisfied, Nash equilibrium play may not necessarily be justified. A long literature explores different ways to weaken the stringent requirements for equilibrium play.

As shown in a growing literature, $p$-dominance is a useful solution concept to study robustness of Nash equilibrium to these deviations. This notion is defined and developed in Morris, Rob, and Shin (1995) and in Kajii and Morris (1997). Consider a probability $p \in[0,1]$. A profile of actions $a^{*}=\left(a_{1}^{*}, a_{2}^{*}, \ldots, a_{I}^{*}\right)$ is a $p$-dominant equilibrium, if for each player $i, a_{i}^{*}$ is a best response of player $i$ to every player $i$ belief that opponents are playing $a_{-i}^{*}$ with probability at least $p$. Morris, Rob, and Shin (1995) show that that in two player, finite action games, for all sufficiently small $p$, a $p$-dominant equilibrium is robust in the sense that it survives iterated deletion of strictly dominated strategies in nearby incomplete information games, extending earlier results on global games due to Carlsson and van Damme (1993) and shedding more light on the equilibrium selection results due to Harsanyi and Selten (1988). Kajii and Morris (1997) show that in finite player, finite action games, for all sufficiently small $p$, a $p$-dominant equilibrium is robust to incomplete information arising from generalized perturbations. Frankel, Morris, and Pauzner (2003) and Hoffmann and Sabarwal (2019) show that in monotone games, $p$-dominant equilibria for sufficiently small $p$ emerge as the unique global games selection, extending the result in Carlsson and van Damme (1993).

Tercieux (2006a) and Tercieux (2006b) have generalized $p$-dominance to the case when players may be uncertain about both the actions being played by opponents and the probability with which different actions are played. Consider a probability $p \in[0,1]$. A collection of sets of actions $S=\left(S_{i}\right)_{i=1}^{I}$, one for each player $i$, is a $p$-best response set ( $p$-BR set), if for each player $i, S_{i}$ contains all best responses of player $i$ when opponents take actions in $S_{-i}$ with probability at least $p$. When every $S_{i}$ is the smallest such set, $S$ is a minimal $p$-best response set ( $p$-MBR set). In the special case when each $S_{i}$ is a singleton, a $p$-MBR set specializes to the case of (strict) $p$-dominant equilibrium. Tercieux (2006a) shows that a $p$-MBR set exists for all $p \in[0,1]$, and $p$-MBR set provides a set valued concept of stability. Moreover, Tercieux (2006b) generalizes the robustness 
results in Kajii and Morris (1997) to p-BR sets and Durieu, Solal, and Tercieux (2011) find that for $p$ sufficiently small, strategies selected by either perturbed joint or independent fictitious play processes must be contained in a unique $p$-MBR set.

In all these papers, the results are of the form that if there is a $p$-dominant equilibrium or a $p$ MBR set with particular properties, then something useful about robustness can be concluded. The question of solving for such $p$-dominant equilibrium or $p$-MBR set is not addressed. Indeed, to the best of the authors' knowledge, there exists no systematic tool to solve for $p$-dominant equilibria or $p$-MBR sets beyond examining each admissible belief that a player may have about her opponents' actions and all admissible supports for these beliefs. As there are uncountably many (a continuum of) such beliefs even in two player, two action games, this can be a very complex task beyond the simplest of cases.

The main contribution of this paper is to show that for monotone games, solving for these robust solution concepts ( $p$-dominant equilibria, exact $p$-BR sets, and $p$-MBR sets) is equivalent to finding particular pure strategy Nash equilibria in a corresponding complete information auxiliary game. The advantages of this approach are threefold. First, traditional methods for finding equilibria may be applied to the complete information auxiliary game, which allows us to solve for robust solution concepts in a straightforward, systematic manner and avoiding the need to consider uncountably many beliefs for each player. Second, as use of Nash equilibrium is ubiquitous, our results make robust solution concepts more accessible to a broader audience, facilitating their broader use. Third, by establishing a bijection between robust solutions in the original game and corresponding Nash equilibria in the auxiliary game, we are able to establish new results on characteristics of entire classes of robust solutions.

A monotone game is one in which each player has either strategic complements or strategic substitutes. This is a general class of games that includes the widely used class of games with strategic complements (or supermodular games), games with strategic substitutes (submodular games), and it includes games in which both types of players are present simultaneously. We allow for finitely many players, and finitely or infinitely many actions (a compact subset of reals). The auxiliary game is formed by taking a "high" copy and a "low" copy of each player in the original game. Thus, if an original game consists of $I$ players, the corresponding auxiliary game will consist of $2 I$ players, so that a strategy profile in the latter can be conveniently described as a pair $(x, y)$, where $x$ is the an $I$-dimensional vector of strategies for the high players, and $y$ is 
the an $I$-dimensional vector of strategies for the low players. The payoff for each high player and each low player is defined in a transparent manner that elicits information about best responses to extremal beliefs and preserves the monotone structure of the original game. In this framework, we show that a profile of actions $a^{*}$ is a $p$-dominant equilibrium in the original game, if, and only if, $\left(a^{*}, a^{*}\right)$ is a Nash equilibrium in the auxiliary game at $p$, and $a^{*}$ is a strict $p$-dominant equilibrium in the original game, if, and only if, $\left(a^{*}, a^{*}\right)$ is a strict Nash equilibrium in the auxiliary game at $p$.

In order to study the corresponding set-valued concepts, we formalize the notion of an exact $p$-best response set ( $p$-EBR set) and the notion of extremal response equilibrium. A $p$-EBR set generalizes the notion of $p$-MBR set and is a special case of $p$-BR set, while extremal response equilibrium generalizes strict Nash equilibrium to allow for particular non-strict Nash equilibria. Characterizations for $p$-EBR sets and $p$-MBR sets are as follows: We show that in a monotone game, if an interval $[y, x]$ of profiles of actions is a nonempty $p$-EBR set in the original game, then $(x, y)$ with $x \geq y$ is an extremal response equilibrium in the auxiliary game. If best responses are interval-valued (Assumption 1), the converse is true as well. This yields a characterization of all $p$-EBR sets in a monotone game. An appropriate specialization yields a characterization of all $p$-MBR sets.

Our characterization yields new theorems for the structure of the entire class of $p$-EBR sets and $p$-MBR sets in monotone games. In games with strategic complements, the class of nonempty $p$-EBR sets and $p$-MBR sets are both complete lattices (in the standard lattice set order). This generalizes the result due to Zhou (1994) for the special case of Nash equilibria (which are $p$-dominant equilibria with $p=1$ ). Minimal deviations from strategic complements destroy this structure completely. If only two players have strict strategic substitutes, or if one player has strict strategic substitutes and another has strict strategic complements, then the class of nonempty, exact $p$-EBR sets and $p$-MBR sets are both totally unordered. This generalizes the result for Nash equilibria in Roy and Sabarwal (2008) and Monaco and Sabarwal (2016). Several examples highlight these results.

The next section presents a motivating example. Section 3 defines the model and presents some results. Section 4 defines and characterizes $p$-dominant equilibrium. Section 5 defines and characterizes $p$-EBR set and $p$-MBR set and gives examples to apply these results. Section 6 presents structure theorems for the class of $p$-EBR sets and $p$-MBR sets. Section 7 concludes. 


\section{Motivating Example}

Example 1. Consider the following two player, three action game with strategic complements. It modifies Example 1 in Tercieux (2006a) so that payoff of each player has increasing differences. Each player has three actions $\{A, B, C\}$ with $A \prec B \prec C$. Payoffs are given in the bimatrix in Figure 1. The game has three Nash equilibria: $(A, A),(B, B)$, and $(C, C)$.

Player 2

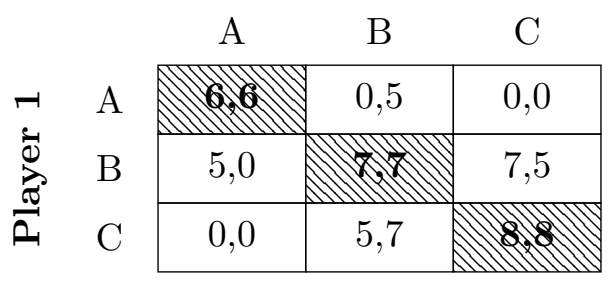

Figure 1: Motivating example

Suppose we are interested in $p$-dominant equilibria for $p=\frac{1}{2}$. (Several results in the literature show that in two player games, $p$-dominant equilibria for $p \leq \frac{1}{2}$ are strongly robust.) The standard way to do this would be to check every belief that puts probability at least $\frac{1}{2}$ on each opponent action and to determine which profiles satisfy the definition. Instead, we consider only two belief-based computations, formalized in the following upper and lower auxiliary games at $p=\frac{1}{2}$.

For the upper auxiliary game at $p=\frac{1}{2}$, player 1 payoff from strategy profile $\left(a_{1}, a_{2}\right)$ is given by weight $p$ on player 1 payoff from $\left(a_{1}, a_{2}\right)$ in the original game and weight $(1-p)$ on player 1 payoff from $\left(a_{1}, C\right)$ in the original game, where $C$ is the highest action of player 2. Player 2 payoff is computed similarly. These payoffs are given in Figure 2. Nash equilibria in the upper auxiliary game are $(B, B)$ and $(C, C)$.

Player 2

\begin{tabular}{|c|c|c|c|}
\hline \multirow{4}{*}{$\begin{array}{l}-1 \\
\dot{\phi} \\
\frac{\vec{\sigma}}{a}\end{array}$} & A & B & $\mathrm{C}$ \\
\hline & 3,3 & 0,6 & 0,4 \\
\hline & 6,0 & 1 & $7,6.5$ \\
\hline & 4,0 & $6.5,7$ & 8 \\
\hline
\end{tabular}

Figure 2: Upper auxiliary game at $p=\frac{1}{2}$

For the lower auxiliary game at $p=\frac{1}{2}$, player 1 payoff from action profile $\left(a_{1}, a_{2}\right)$ is given by 


\section{Player 2}

\begin{tabular}{|c|c|c|c|c|}
\hline \multirow{4}{*}{$\begin{array}{l}-1 \\
\grave{0} \\
\grave{\Xi}\end{array}$} & & A & B & $\mathrm{C}$ \\
\hline & A & 6 & 3,5 & 3,0 \\
\hline & B & 5,3 & 66 & $6,2.5$ \\
\hline & $\mathrm{C}$ & 0,3 & $2.5,6$ & 4,4 \\
\hline
\end{tabular}

Figure 3: Lower auxiliary game at $p=\frac{1}{2}$

weight $p$ on player 1 payoff from $\left(a_{1}, a_{2}\right)$ in the original game and weight $(1-p)$ on player 1 payoff from $\left(a_{1}, A\right)$ in the original game, where $A$ is the lowest action of player 2. Player 2 payoff is computed similarly. These payoffs are given in Figure 3. Nash equilibria in the lower auxiliary game are $(A, A)$ and $(B, B)$.

A special case of our Theorem 2 below is that for a game with strategic complements, a profile $a^{*}$ is a $p$-dominant equilibrium in the original game, if, and only if, $a^{*}$ is an equilibrium in both the upper and the lower auxiliary games at $p$. In this example, only $(B, B)$ is an equilibrium in both the upper and the lower auxiliary games. Therefore, $(B, B)$ is the unique $p$-dominant equilibrium for $p=\frac{1}{2}$. This also shows that $\{B\} \times\{B\}$ is the unique minimal $p$-best response set for $p=\frac{1}{2}$. The other two Nash equilibria are not robust using the criterion of $p$-dominance at $p=\frac{1}{2}$. A similar analysis can be carried out for every $p \in[0,1]$, as shown in Example 3 below.

We extend the logic above to more general cases in Theorems 2, 3, and 4. We show that the upper and lower payoffs at a given $p$ capture all the information needed to evaluate all beliefs that put probability at least $p$ on a given profile of opponent actions $a_{-i}$, and more generally, probability at least $p$ on sets of opponent actions $S_{-i}=\left(S_{j}\right)_{j \neq i}$. Our results apply to games with strategic complements, games with strategic substitutes, and games in which arbitrary numbers of both types of players are present simultaneously. Moreover, the results extend to set-valued concepts and show that all $p$-EBR and $p$-MBR sets can be found in a similar manner.

\section{Monotone game and corresponding auxiliary game}

Consider finite dimensional Euclidean space, $\mathbb{R}^{n}$, with the standard topology and the standard pointwise order, denoted $\leq$. It is a lattice in this order, with lattice operations defined componentwise. For subsets $S$ and $S^{\prime}$ of $\mathbb{R}^{n}, S$ is lower than $S^{\prime}$ in the lattice set order, denoted $S \sqsubseteq S^{\prime}$, 
if for every $x \in S$ and for every $y \in S^{\prime}, x \wedge y \in S$ and $x \vee y \in S^{\prime}{ }^{1}$ Infimum and supremum of a subset $S$ of $\mathbb{R}^{n}$ are denoted $\wedge S$ and $\vee S$, respectively.

Definition 1. A monotone game is a collection $\mathcal{G}=\left(\mathcal{A}_{i}, \pi_{i}\right)_{i \in \mathcal{I}}$, where

1. $\mathcal{I}$ is a finite set of players, $\mathcal{I}=\{1,2, \ldots, I\}$.

2. Each player $i \in \mathcal{I}$ has an action space $\mathcal{A}_{i} \subset \mathbb{R}$ that is a compact sublattice in $\mathbb{R}$. The space of profiles of actions is $\mathcal{A}=\prod_{i \in \mathcal{I}} \mathcal{A}_{i}$. For notational convenience, we use the same symbol $\leq$ to denote the product order on subsets of $\mathcal{A}$.

3. The payoff of each player $i$ is $\pi_{i}: \mathcal{A} \rightarrow \mathbb{R}$. It is continuous and either $\pi_{i}$ has increasing differences in $\left(a_{i}, a_{-i}\right)$, or $\pi_{i}$ has decreasing differences in $\left(a_{i}, a_{-i}\right)$, where, as usual,

- $\pi_{i}$ has increasing differences in $\left(a_{i}, a_{-i}\right)$, if for every $a_{i} \leq a_{i}^{\prime}$ and $a_{-i} \leq a_{-i}^{\prime}$, $\pi_{i}\left(a_{i}^{\prime}, a_{-i}\right)-\pi_{i}\left(a_{i}, a_{-i}\right) \leq \pi_{i}\left(a_{i}^{\prime}, a_{-i}^{\prime}\right)-\pi_{i}\left(a_{i}, a_{-i}^{\prime}\right)$.

- $\pi_{i}$ has decreasing differences in $\left(a_{i}, a_{-i}\right)$, if for every $a_{i} \leq a_{i}^{\prime}$ and $a_{-i} \leq a_{-i}^{\prime}$, $\pi_{i}\left(a_{i}, a_{-i}\right)-\pi_{i}\left(a_{i}^{\prime}, a_{-i}\right) \leq \pi_{i}\left(a_{i}, a_{-i}^{\prime}\right)-\pi_{i}\left(a_{i}^{\prime}, a_{-i}^{\prime}\right)$.

We say that player $i$ is a strategic complements player, if $\pi_{i}$ has increasing differences in $\left(a_{i}, a_{-i}\right)$, and player $i$ is a strategic substitutes player, if $\pi_{i}$ has decreasing differences in $\left(a_{i}, a_{-i}\right)$. The definition of monotone game naturally subsumes games with strategic complements, games with strategic substitutes, and arbitrary combinations of the two. A game with strategic complements (GSC) is a monotone game in which payoff of every player $i$ has increasing differences in $\left(a_{i}, a_{-i}\right)$. A game with strategic substitutes (GSS) is a monotone game in which payoff of every player $i$ has decreasing differences in $\left(a_{i}, a_{-i}\right)$. Given the relation of increasing differences to supermodularity and decreasing differences to submodularity, GSC and GSS are sometimes termed supermodular and submodular games, respectively.

Uncertainty about play of opponents is formalized by a probability measure on actions of opponents. Let $\Delta\left[\mathcal{A}_{-i}\right]$ denote the set of probability measures on the Borel sigma-algebra on $\mathcal{A}_{-i}$. A measure $\mu \in \Delta\left[\mathcal{A}_{-i}\right]$ is viewed as player $i$ 's belief about play of opponents. Player $i$ 's expected payoff from playing $a_{i} \in \mathcal{A}_{i}$ when belief about play of opponents is $\mu \in \Delta\left[\mathcal{A}_{-i}\right]$ is

$$
\pi_{i}\left(a_{i}, \mu\right)=\int_{\mathcal{A}_{-i}} \pi_{i}\left(a_{i}, a_{-i}\right) d \mu
$$

\footnotetext{
${ }^{1}$ The lattice set order is due to Veinott (1989), as mentioned in Topkis (1978). Other terms used in the literature are induced set ordering and strong set order.
} 
Following Morris, Rob, and Shin (1995) and Kajii and Morris (1997), p-dominant equilibrium is defined as follows. For each $p \in[0,1]$, a profile of actions $a^{*} \in \mathcal{A}$ is a $p$-dominant equilibrium, if for every player $i \in \mathcal{I}, a_{i}^{*}$ is a best response of player $i$ whenever player $i$ believes that opponents will play $a_{-i}^{*}$ with probability at least $p$. That is, for every player $i$,

$$
a_{i}^{*} \in\left\{a_{i} \in \mathcal{A}_{i} \mid \forall \mu \in \Delta\left[\mathcal{A}_{-i}\right] \text { with } \mu\left(a_{-i}^{*}\right) \geq p, \forall a_{i}^{\prime} \in \mathcal{A}_{i}, \pi_{i}\left(a_{i}, \mu\right) \geq \pi_{i}\left(a_{i}^{\prime}, \mu\right)\right\}
$$

Following Tercieux (2006a), a $p$-best response set and minimal $p$-best response set are defined as follows. For each player $i$, consider measurable $S_{i} \subseteq \mathcal{A}_{i}$, let $S=\prod_{i \in \mathcal{I}} S_{i}$ and $S_{-i}=\prod_{j \neq i} S_{j}$. For each $p \in[0,1]$, a $p$-belief for player $i$ that opponents play in $S_{-i}$ is a probability measure $\mu \in \Delta\left[\mathcal{A}_{-i}\right]$ that assigns to $S_{-i}$ probability at least $p$. Let $M^{p}\left[S_{-i}\right]$ denote the set of all such probability measures, that is,

$$
M^{p}\left[S_{-i}\right]=\left\{\mu \in \Delta\left[\mathcal{A}_{-i}\right] \mid \mu\left(S_{-i}\right) \geq p\right\}
$$

When each $S_{i} \subseteq \mathcal{A}_{i}$ is nonempty and compact, let $\Lambda_{i}\left[S_{-i}, p\right]$ be the set of player $i$ 's best responses when player $i$ believes that opponents will play in $S_{-i}$ with probability at least $p$, that is,

$$
\Lambda_{i}\left[S_{-i}, p\right]=\left\{a_{i} \in \mathcal{A}_{i} \mid \exists \mu \in M^{p}\left[S_{-i}\right], \forall a_{i}^{\prime} \in \mathcal{A}_{i}, \pi_{i}\left(a_{i}, \mu\right) \geq \pi_{i}\left(a_{i}^{\prime}, \mu\right)\right\} .
$$

Moreover, let $\Lambda[S, p]=\prod_{i \in \mathcal{I}} \Lambda_{i}\left[S_{-i}, p\right]$. A set $S=\prod_{i \in \mathcal{I}} S_{i}$ is a $p$-best response set, or $p$-BR set, if $\Lambda[S, p] \subseteq S$. A set $S$ is a minimal $p$-best response set, or $p$-MBR set, if $S$ is a $p$-BR set and $S$ does not contain any proper subset that is a $p$-BR set. The following properties of $p$-MBR sets due to Tercieux (2006a) are useful for the analysis here.

Proposition 1. (Tercieux, 2006a) For each fixed $p \in[0,1]$,

1. Every game has a $p$-MBR set.

2. Two distinct $p$-MBR sets are disjoint.

3. If $S$ is a $p$-MBR set, then $\Lambda[S, p]=S$.

In developing the results here, an important insight is that in a monotone game, for each $p \in[0,1]$, we can construct bounds on measures in $M^{p}\left[S_{-i}\right]$ and on best responses to those measures 
as follows. For each $p \in[0,1]$ and each $z_{-i} \in \mathcal{A}_{-i}$, let $\underline{\mu}_{z_{-i}}, \bar{\mu}_{z_{-i}} \in \Delta\left[\mathcal{A}_{-i}\right]$ be defined as

$$
\underline{\mu}_{z_{-i}}=p \delta_{z_{-i}}+(1-p) \delta_{\wedge \mathcal{A}_{-i}} \quad \text { and } \quad \bar{\mu}_{z_{-i}}=p \delta_{z_{-i}}+(1-p) \delta_{\vee \mathcal{A}_{-i}}
$$

where, as usual, $\delta_{a}$ is the degenerate measure that puts probability one on $a, \wedge \mathcal{A}_{-i}=\inf \mathcal{A}_{-i}$, and $\vee \mathcal{A}_{-i}=\sup \mathcal{A}_{-i}$. The measure $\underline{\mu}_{z_{-i}}$ puts probability $p$ on $z_{-i}$ and probability $1-p$ on the lowest profile of opponent actions, and measure $\underline{\mu}_{z_{-i}}$ puts probability $p$ on $z_{-i}$ and probability $1-p$ on the highest profile of opponent actions. Payoff to player $i$ from playing $x_{i}$ when opponents are playing $\underline{\mu}_{z_{-i}}$ or $\bar{\mu}_{z_{-i}}$ is

$$
\begin{aligned}
& \pi_{i}\left(x_{i}, \underline{\mu}_{z_{-i}}\right)=p \pi_{i}\left(x_{i}, z_{-i}\right)+(1-p) \pi_{i}\left(x_{i}, \wedge \mathcal{A}_{-i}\right) \\
& \pi_{i}\left(x_{i}, \bar{\mu}_{z_{-i}}\right)=p \pi_{i}\left(x_{i}, z_{-i}\right)+(1-p) \pi_{i}\left(x_{i}, \vee \mathcal{A}_{-i}\right)
\end{aligned}
$$

The intuition that $\underline{\mu}_{z_{-i}}$ is the smallest measure that puts probability $p$ on $z_{-i}$ and $\bar{\mu}_{z_{-i}}$ is the largest is formalized using first order stochastic dominance. Recall that for $\mu^{\prime}, \mu \in \Delta\left[\mathcal{A}_{-i}\right]$, $\mu^{\prime}$ first order stochastically dominates $\mu$, denoted $\mu \leq_{F} \mu^{\prime}$, if for every increasing set $E \subseteq \mathcal{A}_{-i}$, $\mu(E) \leq \mu^{\prime}(E)$. As usual, a set $E \subseteq \mathcal{A}_{-i}$ is increasing if for each $x \in E$ and each $y \in \mathcal{A}_{-i}$, $x \leq y \Rightarrow y \in E$. Measures in $M^{p}\left[S_{-i}\right]$ and best responses to such measures are bounded as follows.

Lemma 1. Let $i \in \mathcal{I}, S_{-i}=\prod_{j \neq i} S_{j}$, and $p \in[0,1]$. For every $\mu \in M^{p}\left[S_{-i}\right]$,

1. $\underline{\mu}_{\wedge S_{-i}} \leq_{F} \quad \mu \quad \leq_{F} \quad \bar{\mu}_{\vee S_{-i}}$

2. If $i \in \mathcal{I}$ is a strategic complements player, then

$$
B R_{i}\left(\underline{\mu}_{\wedge S_{-i}}\right) \sqsubseteq B R_{i}(\mu) \sqsubseteq B R_{i}\left(\bar{\mu}_{\vee S_{-i}}\right)
$$

3. If $i \in \mathcal{I}$ is a strategic substitutes player, then

$$
B R_{i}\left(\bar{\mu}_{\vee S_{-i}}\right) \sqsubseteq B R_{i}(\mu) \sqsubseteq B R_{i}\left(\underline{\mu}_{\wedge S_{-i}}\right) .
$$

Proof. For statement (1), let $\mu \in M^{p}\left[S_{-i}\right]$ and $E \subseteq \mathcal{A}_{-i}$ be increasing. If $S_{-i} \cap E \neq \emptyset$, then for every $x \in S_{-i} \cap E, x \leq \vee S_{-i} \leq \vee \mathcal{A}_{-i}$, whence $\bar{\mu}_{\vee S_{-i}}(E)=p+(1-p)=1 \geq \mu(E)$. If $S_{-i} \cap E=\emptyset$, then as $E$ is increasing, $\bar{\mu}_{\vee S_{-i}}(E) \geq 1-p$, and moreover, it must be that $\mu(E) \leq 1-p$, because if $\mu(E)>1-p$, then $\mu\left(S_{-i}\right)+\mu(E)>p+(1-p)=1$, a contradiction. Thus, $\mu(E) \leq 1-p \leq \bar{\mu}_{\vee S_{-i}}(E)$, whence $\mu \leq_{F} \bar{\mu}_{\vee S_{-i}}$. Similarly, it can be shown that $\underline{\mu}_{\wedge S_{-i}} \leq_{F} \mu$. 
For statement (2), notice that for $\hat{x}_{i}, \tilde{x}_{i} \in \mathcal{A}_{i}$,

$$
\pi_{i}\left(\tilde{x}_{i}, \mu\right)-\pi_{i}\left(\hat{x}_{i}, \mu\right)=\int_{\mathcal{A}_{-i}} \pi_{i}\left(\tilde{x}_{i}, x_{-i}\right)-\pi_{i}\left(\hat{x}_{i}, x_{-i}\right) d \mu\left(x_{-i}\right)
$$

If player $i$ is a strategic complements player, then $\pi_{i}\left(x_{i}, x_{-i}\right)$ has increasing differences in $\left(x_{i}, x_{-i}\right)$, and therefore, $\pi_{i}\left(x_{i}, \mu\right)$ has increasing differences in $\left(x_{i}, \mu\right)$, where the partial order on distributions is given by first order stochastic dominance. Consequently, by Topkis (1978), $B R_{i}\left(\underline{\mu}_{\wedge S_{-i}}\right) \sqsubseteq$ $B R_{i}(\mu) \sqsubseteq B R_{i}\left(\bar{\mu}_{\vee S_{-i}}\right)$. Statement (3) follows similarly.

We analyze $p$-dominance and $p$-MBR sets using an auxiliary game defined as follows.

Definition 2. Let $\mathcal{G}=\left(\mathcal{A}_{i}, \pi_{i}\right)_{i \in \mathcal{I}}$ be a monotone game. The auxiliary game at $p \in[0,1]$ is $\widetilde{\mathcal{G}}=\left(\widetilde{\mathcal{A}}_{i}, \widetilde{\pi}_{i}\right)_{i \in \tilde{\mathcal{I}}}$, where

1. $\widetilde{\mathcal{I}}=\mathcal{I}_{H} \cup \mathcal{I}_{L}$ is a set of $2 I$ players, two copies (one high, one low) for each player in $\mathcal{G}$. The high players are denoted by $\mathcal{I}_{H}=\left\{1_{H}, \ldots, i_{H}, \ldots, I_{H}\right\}$ and the low players by $\mathcal{I}_{L}=$ $\left\{1_{L}, \ldots, i_{L}, \ldots, I_{L}\right\}$.

2. The action space for each $i_{H} \in \mathcal{I}_{H}$ and $i_{L} \in \mathcal{I}_{L}$ is the same as that for the corresponding player $i$ in $\mathcal{G}$. That is, $\widetilde{\mathcal{A}}_{i_{H}}=\widetilde{\mathcal{A}}_{i_{L}}=\mathcal{A}_{i}$, with the same Euclidean order. The space of profiles of actions is $\widetilde{\mathcal{A}}=\mathcal{A}_{H} \times \mathcal{A}_{L}$, where $\mathcal{A}_{H}=\prod_{i \in \mathcal{I}_{H}} \widetilde{\mathcal{A}}_{i_{H}}$ and $\mathcal{A}_{L}=\prod_{i \in \mathcal{I}_{L}} \widetilde{\mathcal{A}}_{i_{L}}$. When convenient, profiles $x, y \in \mathcal{A}$ are identified naturally with profile $(x, y) \in \mathcal{A}_{H} \times \mathcal{A}_{L}$, and conversely.

3. Payoffs are defined as follows.

- If $i \in \mathcal{I}$ has increasing differences in $\left(a_{i}, a_{-i}\right)$, then for the corresponding $i_{H}$ and $i_{L}$,

$$
\tilde{\pi}_{i_{H}}(x, y)=\pi_{i}\left(x_{i}, \bar{\mu}_{x_{-i}}\right) \quad \text { and } \quad \tilde{\pi}_{i_{L}}(x, y)=\pi_{i}\left(y_{i}, \underline{\mu}_{y_{-i}}\right) .
$$

- If $i \in \mathcal{I}$ has decreasing differences in $\left(a_{i}, a_{-i}\right)$, then for the corresponding $i_{H}$ and $i_{L}$,

$$
\tilde{\pi}_{i_{H}}(x, y)=\pi_{i}\left(x_{i}, \underline{\mu}_{y_{-i}}\right) \quad \text { and } \quad \tilde{\pi}_{i_{L}}(x, y)=\pi_{i}\left(y_{i}, \bar{\mu}_{x_{-i}}\right) .
$$

The auxiliary game has the following features. Although there are a total of $2 I$ players in the auxiliary game, each player has $I-1$ opponents. The identity of the opponents depends on whether a player has strategic complements in the original game or strategic substitutes. If player $i \in \mathcal{I}$ is 
a strategic complements player, then payoff for its high copy $i_{H}$ in $\widetilde{\mathcal{G}}$ is affected only by high copies of other players, and payoff for its low copy $i_{L}$ in $\widetilde{\mathcal{G}}$ is affected only by low copies of other players. If player $i \in \mathcal{I}$ is a strategic substitutes player, then payoff for its high copy $i_{H}$ is affected only by low copies of other players, and payoff for its low copy $i_{L}$ is affected only by high copies of other players.

The construction of the auxiliary game preserves strategic complements and strategic substitutes for each player.

Theorem 1. Let $\mathcal{G}$ be a monotone game and $\widetilde{\mathcal{G}}$ the auxiliary game at $p \in[0,1]$.

1. If $i \in \mathcal{I}$ is a strategic complements player in $\mathcal{G}$, then both $i_{H}$ and $i_{L}$ are strategic complements players in $\widetilde{\mathcal{G}}$.

2. If $i \in \mathcal{I}$ is a strategic substitutes player in $\mathcal{G}$, then both $i_{H}$ and $i_{L}$ are strategic substitutes players in $\widetilde{\mathcal{G}}$.

3. $\widetilde{\mathcal{G}}$ is a monotone game.

Proof. For statement (1), suppose player $i$ is a strategic complements player in $\mathcal{G}$. Then payoff for its high copy in $\widetilde{\mathcal{G}}$ is given by

$$
\tilde{\pi}_{i_{H}}(x, y)=\pi_{i}\left(x_{i}, \bar{\mu}_{x_{-i}}\right)=p \pi_{i}\left(x_{i}, x_{-i}\right)+(1-p) \pi_{i}\left(x_{i}, \vee \mathcal{A}_{-i}\right)
$$

If $p=0$, then $\widetilde{\pi}_{i_{H}}$ is constant with respect to opponent actions $(x, y)_{-i_{H}}$, and therefore, $\widetilde{\pi}_{i_{H}}$ satisfies increasing differences in $\left(x_{i_{H}},(x, y)_{-i_{H}}\right)$ trivially. For $p>0, \widetilde{\pi}_{i_{H}}$ does not depend on $y$, and therefore, $\tilde{\pi}_{i_{H}}$ satisfies increasing differences in $\left(x_{i_{H}},(x, y)_{-i_{H}}\right)$, if, and only if, $\pi_{i}$ satisfies increasing differences in $\left(x_{i}, x_{-i}\right)$. This shows that $i_{H}$ is a strategic complements player in $\widetilde{\mathcal{G}}$. A similar argument shows that $i_{L}$ is a strategic complements player in $\widetilde{\mathcal{G}}$. Statement $(2)$ is proved similarly. Statement (3) follows from statements (1) and (2).

Payoff functions in the auxiliary game are designed to produce the highest and lowest best response for each type of player, in the following sense.

If player $i$ is a strategic complements player, then payoff for its high copy is given by $\tilde{\pi}_{i_{H}}(x, y)=$ $\pi_{i}\left(x_{i}, \bar{\mu}_{x_{-i}}\right)$, and therefore, best response of its high copy is given by $\widetilde{B R}_{i_{H}}\left((x, y)_{-i_{H}}\right)=B R_{i}\left(\bar{\mu}_{x_{-i}}\right)$, where $B R_{i}\left(\bar{\mu}_{x_{-i}}\right)$ is best response of player $i$ to belief $\bar{\mu}_{x_{-i}}$ about opponent actions in the original 
game $\mathcal{G}$. Lemma 1 shows that if $S_{-i}=\left\{x_{-i}\right\}$, then $\bar{\mu}_{x_{-i}}$ is the highest belief that opponents play $x_{-i}$ with probability at least $p$. As player $i$ is a strategic complements player, Lemma 1 shows further that $B R_{i}\left(\bar{\mu}_{x_{-i}}\right)$ is the highest best response. Similarly, payoff for its low copy is $\widetilde{\pi}_{i_{L}}(x, y)=$ $\pi_{i}\left(y_{i}, \underline{\mu}_{y_{-i}}\right)$, and therefore, best response for its low copy is $\widetilde{B R}_{i_{L}}\left((x, y)_{-i_{L}}\right)=B R_{i}\left(\underline{\mu}_{y_{-i}}\right)$, where $B R_{i}\left(\underline{\mu}_{y_{-i}}\right)$ is best response of player $i$ to belief $\underline{\mu}_{y_{-i}}$ about opponent actions in the original game $\mathcal{G}$. Lemma 1 shows that $\underline{\mu}_{y_{-i}}$ is the lowest belief that opponents play $y_{-i}$ with probability at least $p$. As player $i$ is a strategic complements player, Lemma 1 shows further that $B R_{i}\left(\underline{\mu}_{y_{-i}}\right)$ is the lowest best response. To summarize, if player $i$ is a strategic complements player, then

$$
\widetilde{B R}_{i_{H}}\left((x, y)_{-i_{H}}\right)=B R_{i}\left(\bar{\mu}_{x_{-i}}\right) \text { and } \widetilde{B R}_{i_{L}}\left((x, y)_{-i_{L}}\right)=B R_{i}\left(\underline{\mu}_{y_{-i}}\right)
$$

A similar argument shows that if player $i$ is a strategic substitutes player, then

$$
\widetilde{B R}_{i_{H}}\left((x, y)_{-i_{H}}\right)=B R_{i}\left(\underline{\mu}_{y_{-i}}\right) \text { and } \widetilde{B R}_{i_{L}}\left((x, y)_{-i_{L}}\right)=B R_{i}\left(\bar{\mu}_{x_{-i}}\right)
$$

The definition of (pure strategy) Nash equilibrium is unchanged, both in the original game and in the auxiliary game.

In the special case when all players are strategic complements players, that is, for a GSC, there is an additional aspect of the auxiliary game that is useful. In this case, the definition shows that for each player, payoff for their high copy is affected by actions of high types of other players only, and payoff for their low copy is affected by actions of low types of other players only. This implies that the auxiliary game $\widetilde{\mathcal{G}}$, which is also a GSC, can be decomposed into two GSC, an upper auxiliary game, denoted $\widetilde{\mathcal{G}}_{H}$, and a lower auxiliary game, denoted $\widetilde{\mathcal{G}}_{L}$. In $\widetilde{\mathcal{G}}_{H}$, only high copies of all players are included, and in $\widetilde{\mathcal{G}}_{L}$, only low copies of all players are included. In particular, a profile $(x, y)$ is a Nash equilibrium in $\widetilde{\mathcal{G}}$, if, and only if, $x$ is a Nash equilibrium in $\widetilde{\mathcal{G}}_{H}$ and $y$ is a Nash equilibrium

in $\widetilde{\mathcal{G}}_{L}$. This is helpful to analyze GSC. This decomposition does not hold either for general GSS or for games in which both types of players are present.

\section{Characterizing $p$-dominant equilibrium}

The main result here characterizes $p$-dominant and strict $p$-dominant equilibrium in terms of Nash equilibrium in the corresponding auxiliary game. For each $p \in[0,1]$, a profile of actions $a^{*} \in \mathcal{A}$ is 
a $p$-dominant equilibrium, if for every player $i \in \mathcal{I}$,

$$
a_{i}^{*} \in\left\{a_{i} \in \mathcal{A}_{i} \mid \forall \mu \in M^{p}\left[\left\{a_{-i}^{*}\right\}\right], \forall a_{i}^{\prime} \in \mathcal{A}_{i}, \pi_{i}\left(a_{i}, \mu\right) \geq \pi_{i}\left(a_{i}^{\prime}, \mu\right)\right\} .
$$

We also define the notion of strict $p$-dominant equilibrium. For each $p \in[0,1]$, a profile of actions $a^{*} \in \mathcal{A}$ is a strict $p$-dominant equilibrium, if for every player $i \in \mathcal{I}$,

$$
a_{i}^{*} \in\left\{a_{i} \in \mathcal{A}_{i} \mid \forall \mu \in M^{p}\left[\left\{a_{-i}^{*}\right\}\right], \forall a_{i}^{\prime} \in \mathcal{A}_{i} \backslash\left\{a_{i}\right\}, \pi_{i}\left(a_{i}, \mu\right)>\pi_{i}\left(a_{i}^{\prime}, \mu\right)\right\} .
$$

The strength of Theorem 2 below is that in order to locate every $p$-dominant equilibrium and every strict $p$-dominant equilibrium, it is both necessary and sufficient to consider a subset of pure strategy Nash equilibria in the auxiliary game. In general, locating $p$-dominant equilibria requires checking the best response of every player to every possible belief that puts probability $p$ or more on different profiles of opponent actions. This involves taking into account uncountably many beliefs even in simple cases when players have only a few actions. Construction of the auxiliary game makes the task much easier by considering high and low payoffs only and by looking for particular Nash equilibria.

In other words, $p$-dominant equilibrium and strict $p$-dominant equilibrium in the original game may be found with the same tractability as that of computing pure strategy Nash equilibrium in the auxiliary game. This is much easier given the availability of an array of optimization, computation, and fixed point techniques to find Nash equilibrium in a game.

Theorem 2. Let $\mathcal{G}$ be a monotone game and $\widetilde{\mathcal{G}}$ the auxiliary game at $p \in[0,1]$.

1. A profile of actions $a^{*}$ is a p-dominant equilibrium in $\mathcal{G}$, if, and only if, $\left(a^{*}, a^{*}\right)$ is a Nash equilibrium in $\widetilde{\mathcal{G}}$.

2. A profile of actions $a^{*}$ is a strict $p$-dominant equilibrium in $\mathcal{G}$, if, and only if, $\left(a^{*}, a^{*}\right)$ is a strict Nash equilibrium in $\widetilde{\mathcal{G}}$.

Proof. Consider statement (1). Suppose $a^{*}$ is a $p$-dominant equilibrium in $\mathcal{G}$. Then for every player $i$ and for every $\mu \in M^{p}\left(\left\{a_{-i}^{*}\right\}\right), a_{i}^{*} \in B R_{i}(\mu)$. In particular, $a_{i}^{*} \in B R_{i}\left(\bar{\mu}_{a_{-i}^{*}}\right)$ and $a_{i}^{*} \in B R_{i}\left(\underline{\mu}_{a_{-i}^{*}}\right)$. Suppose player $i$ is a strategic complements player and consider its two copies $i_{H}$ and $i_{L}$ in $\widetilde{\mathcal{G}}$. Then $a_{i}^{*} \in B R_{i}\left(\bar{\mu}_{a_{-i}^{*}}\right)=\widetilde{B R}_{i_{H}}\left(\left(a^{*}, a^{*}\right)_{-i_{H}}\right)$ and $a_{i}^{*} \in B R_{i}\left(\underline{\mu}_{a_{-i}^{*}}\right)=\widetilde{B R}_{i_{L}}\left(\left(a^{*}, a^{*}\right)_{-i_{L}}\right)$. A similar 
argument works if player $i$ is a strategic substitutes player. Thus, $\left(a^{*}, a^{*}\right)$ is a Nash equilibrium in $\widetilde{\mathcal{G}}$. In the other direction, suppose $\left(a^{*}, a^{*}\right)$ is a Nash equilibrium in $\widetilde{\mathcal{G}}$. Fix player $i \in \mathcal{I}$, and let $a_{i}^{*}$ be the common value of $a_{i_{H}}^{*}$ and $a_{i_{L}}^{*}$. If $i$ is a strategic complements player, then for every $\mu \in M^{p}\left(\left\{a_{-i}^{*}\right\}\right)$

$$
B R_{i}\left(\underline{\mu}_{a_{-i}^{*}}\right) \sqsubseteq B R_{i}(\mu) \sqsubseteq B R_{i}\left(\bar{\mu}_{a_{-i}^{*}}\right) .
$$

Moreover, $\left(a^{*}, a^{*}\right)$ is a Nash equilibrium implies that $a_{i}^{*} \in \widetilde{B R}_{i_{H}}\left(\left(a^{*}, a^{*}\right)_{-i_{H}}\right)=B R_{i}\left(\bar{\mu}_{a_{-i}^{*}}\right)$ and $a_{i}^{*} \in \widetilde{B R}_{i_{L}}\left(\left(a^{*}, a^{*}\right)_{-i_{L}}\right)=B R_{i}\left(\underline{\mu}_{a_{-i}^{*}}\right)$. As $B R_{i}(\mu) \neq \emptyset$, pick $b \in B R_{i}(\mu)$, and then properties of lattice set order imply that $a_{i}^{*}=\left(a_{i}^{*} \vee b\right) \wedge a_{i}^{*} \in B R_{i}(\mu)$. A similar argument works if $i$ is a strategic substitutes player. Thus, for every $i$ and for every $\mu \in M^{p}\left(\left\{a_{-i}^{*}\right\}\right), a_{i}^{*} \in B R_{i}(\mu)$, whence $a^{*}$ is a p-dominant equilibrium in $\mathcal{G}$.

Consider statement (2). Suppose $a^{*}$ is a strict $p$-dominant equilibrium. Then for every player $i$ and for every $\mu \in M^{p}\left(\left\{a_{-i}^{*}\right\}\right), a_{i}^{*}=B R_{i}(\mu)$. In particular, $a_{i}^{*}=B R_{i}\left(\bar{\mu}_{a_{-i}^{*}}\right)$ and $a_{i}^{*}=B R_{i}\left(\underline{\mu}_{a_{-i}^{*}}\right)$. Suppose player $i$ is a strategic complements player and consider its two copies $i_{H}$ and $i_{L}$ in $\widetilde{\mathcal{G}}$. Then $a_{i}^{*}=B R_{i}\left(\bar{\mu}_{a_{-i}^{*}}\right)=\widetilde{B R}_{i_{H}}\left(\left(a^{*}, a^{*}\right)_{-i_{H}}\right)$ and $a_{i}^{*}=B R_{i}\left(\underline{\mu}_{a_{-i}^{*}}\right)=\widetilde{B R}_{i_{L}}\left(\left(a^{*}, a^{*}\right)_{-i_{L}}\right)$. A similar argument works if $i$ is a strategic substitutes player. Consequently, $\left(a^{*}, a^{*}\right)$ is a strict Nash equilibrium in $\widetilde{\mathcal{G}}$. In the other direction, suppose $\left(a^{*}, a^{*}\right)$ is a strict Nash equilibrium in $\widetilde{\mathcal{G}}$. Fix player $i \in \mathcal{I}$, and let $a_{i}^{*}$ be the common value of $a_{i_{H}}^{*}$ and $a_{i_{L}}^{*}$. If $i$ is a strategic complements player, then for every $\mu \in M^{p}\left(\left\{a_{-i}^{*}\right\}\right)$,

$$
\left\{a_{i}^{*}\right\} \sqsubseteq B R_{i}(\mu) \sqsubseteq\left\{a_{i}^{*}\right\}
$$

whence $B R_{i}(\mu)=a_{i}^{*}$. A similar argument works if player $i$ is a strategic substitutes player. Thus, $a^{*}$ is a strict $p$-dominant equilibrium in $\mathcal{G}$.

Theorem 2 provides a natural bijection between $p$-dominant equilibria in $\mathcal{G}$ and particular Nash equilibria in $\widetilde{\mathcal{G}}$, and between strict $p$-dominant equilibria in $\mathcal{G}$ and particular strict Nash equilibria in $\widetilde{\mathcal{G}}$. Moreover, it helps to characterize singleton $p$-MBR sets, as follows.

Corollary 1. Let $\mathcal{G}$ be a monotone game and $\widetilde{\mathcal{G}}$ the auxiliary game at $p \in[0,1]$.

For a profile of actions $a^{*} \in \mathcal{A}$, $\left\{a^{*}\right\}$ is a $p-M B R$ set in $\mathcal{G}$, if, and only if, $\left(a^{*}, a^{*}\right)$ is a strict Nash equilibrium in $\widetilde{\mathcal{G}}$.

Proof. Tercieux (2006a) points out that $\left\{a^{*}\right\}$ is a singleton $p$-MBR set, if, and only if, $a^{*}$ is a strict $p$-dominant equilibrium, and Theorem 2 shows that this is equivalent to $\left(a^{*}, a^{*}\right)$ is a strict Nash 
equilibrium in $\widetilde{\mathcal{G}}$.

\section{$5 \quad$ Characterizing $p$-EBR set and $p$-MBR set}

In order to characterize $p$-MBR sets more generally, it is useful to consider a new class of sets. A set $S=\prod_{i \in \mathcal{I}} S_{i}$ is an exact $p$-best response set, denoted $p$-EBR set, if $\Lambda(S, p)=S$. In other words, for every player $i, \Lambda_{i}\left(S_{-i}, p\right)=S_{i}$. As shown in proposition 1 , every $p$-MBR set has this property, and therefore, $p$-EBR set is a generalization of $p$-MBR set. Moreover, it is immediate that every $p$-EBR set is a $p$-BR set. The converse to both statements is not necessarily true. In other words, $p$-EBR set is an intermediate notion between $p$-MBR set and $p$-BR set.

We shall also find it useful to formalize a notion of extremal response equilibrium in the auxiliary game. Let $\mathcal{G}$ be a monotone game and $\widetilde{\mathcal{G}}$ the auxiliary game at $p \in[0,1]$. A Nash equilibrium $(x, y)$ in $\widetilde{\mathcal{G}}$ is an extremal response equilibrium, if all high players are best responding with their highest best response, and all low players are best responding with their lowest best response. That is, for every $i_{H} \in \mathcal{I}_{H}, x_{i_{H}}=\vee \widetilde{B R}_{i_{H}}\left((x, y)_{-i_{H}}\right)$, and for every $i_{L} \in \mathcal{I}_{L}, y_{i_{L}}=\wedge \widetilde{B R}_{i_{L}}\left((x, y)_{-i_{L}}\right)$. Notice that every strict Nash equilibrium is trivially an extremal response equilibrium. The definition generalizes this to the case when a player may have multiple best responses in equilibrium, in which case the extremal response equilibrium is the one in which high players are playing their highest best response and low players are playing their lowest best response.

As shown in Theorem 3, extremal response equilibrium emerges naturally as a necessary condition to study $p$-EBR sets. For it to be a sufficient condition, the following assumption is helpful.

Assumption 1. For each player $i \in \mathcal{I}$, each $p \in[0,1]$, and each $a_{-i}, a_{-i}^{\prime} \in \mathcal{A}_{-i}$ such that $a_{-i}^{\prime} \geq a_{-i}$,

$$
B R_{i}\left(p \delta_{a_{-i}}+(1-p) \delta_{a_{-i}^{\prime}}\right)
$$

is interval-valued.

When $\mathcal{A}_{i}$ is an interval in $\mathbb{R}$, assumption 1 is equivalent to the statement that $B R_{i}\left(p \delta_{a_{-i}}+\right.$ $\left.(1-p) \delta_{a_{-i}^{\prime}}\right)$ is convex valued, for which any one of the following conditions is sufficient. Consider the corresponding payoff function $p \pi_{i}\left(\cdot, a_{-i}\right)+(1-p) \pi_{i}\left(\cdot, a_{-i}^{\prime}\right)$ for which $B R_{i}\left(p \delta_{a_{-i}}+(1-p) \delta_{a_{-i}^{\prime}}\right)$ is the best response. One sufficient condition for Assumption 1 to be satisfied is that this payoff function has a unique maximizer, in which case it is trivially convex valued. A standard sufficient 
condition is that the payoff function is strictly quasiconcave. ${ }^{2}$ A second sufficient condition is that each $\pi_{i}\left(\cdot, a_{-i}\right)$ is concave in $a_{i}$, in which case the payoff function is concave, and therefore, the best response is convex valued. For a third sufficient condition, Choi and Smith (2017) give conditions under which the sum of two quasiconcave functions remains quasiconcave, so that if each $\pi_{i}\left(\cdot, a_{-i}\right)$ is quasiconcave in $a_{i}$ and has an increasing portion that is more concave than each decreasing portion, their weighted sum is quasiconcave in $a_{i}$ as well, and this implies that best response is convex valued.

When $\mathcal{A}_{i} \subseteq \mathbb{R}$ is not convex, another sufficient condition based on diminishing returns is available. Say that $\pi_{i}$ has diminishing returns in $a_{i}$, if for every $a_{-i} \in \mathcal{A}_{-i}$, and every $a_{i}^{\prime \prime}, a_{i}^{\prime}, a_{i} \in \mathcal{A}_{i}$ with $a_{i}^{\prime \prime}>a_{i}^{\prime}>a_{i}$

$$
\pi\left(a_{i}^{\prime}, a_{-i}\right)-\pi\left(a_{i}, a_{-i}\right) \geq \pi\left(a_{i}^{\prime \prime}, a_{-i}\right)-\pi\left(a_{i}^{\prime}, a_{-i}\right) .
$$

In other words, for fixed actions of opponents $a_{-i}$, the marginal return to player $i$ from playing $a_{i}^{\prime}$ over a lower $a_{i}$ are reduced when player $i$ plays the higher pair $a_{i}^{\prime \prime}$ versus $a_{i}^{\prime}$. Morris and Ui (2005) use this assumption with strategic complements. Sufficiency of this condition is formalized in the following proposition.

Proposition 2. If for every player $i \in \mathcal{I}, \pi_{i}$ has diminishing returns in $a_{i}$, then Assumption 1 is satisfied.

Proof. See Appendix.

Lemma 2. Let $\mathcal{G}$ be a monotone game, $\widetilde{\mathcal{G}}$ the auxiliary game at $p \in[0,1]$, and suppose Assumption 1 holds. If $(x, y)$ is an extremal response equilibrium in $\widetilde{\mathcal{G}}$ with $x \geq y$, then for every $i \in \mathcal{I}$, $\left[y_{i}, x_{i}\right] \subseteq \Lambda_{i}\left(\left\{y_{-i}, x_{-i}\right\}, p\right)$

Proof. See Appendix.

The next theorem gives the main result in this section. It is significant because solving for all $p$-MBR sets directly from the definition in Tercieux (2006a) involves evaluating each player's best responses to all beliefs which put at least probability $p$ over arbitrary subsets of opponents' actions. This is a daunting task even with few players and few actions. Our approach reduces

\footnotetext{
${ }^{2}$ See Christensen (2017) for necessary and sufficient conditions for when a function has a unique maximizer.
} 
this problem to checking for extremal response equilibria in the auxiliary game. Indeed, Theorem 3 shows that in order to find $p$-EBR sets (a class that includes $p$-MBR sets), it is necessary and sufficient to consider extremal response equilibria in the auxiliary game. We don't need to evaluate best responses to uncountably many beliefs each time over different subsets of opponent actions. Consequently, $p$-EBR sets (and therefore, $p$-MBR sets) can be found using standard techniques to compute Nash equilibrium.

Theorem 3. Let $\mathcal{G}$ be a monotone game and $\widetilde{\mathcal{G}}$ the auxiliary game at $p \in[0,1]$.

1. If $S$ is a nonempty $p-E B R$ set in $\mathcal{G}$, then $(\vee S, \wedge S)$ is an extremal response equilibrium in $\widetilde{\mathcal{G}}$.

2. If $(x, y)$ is an extremal response equilibrium in $\widetilde{\mathcal{G}}$ with $x \geq y$, then the interval $[y, x]$ is a nonempty $p-B R$ set in $\mathcal{G}$.

3. Suppose Assumption 1 is satisfied.

(a) Interval $[y, x]$ is a nonempty $p$-EBR set in $\mathcal{G}$, if, and only if, profile $(x, y)$ with $x \geq y$ is an extremal response equilibrium in $\widetilde{\mathcal{G}}$.

(b) Every nonempty $p$-EBR set $S$ in $\mathcal{G}$ is an interval of the form $S=[\wedge S, \vee S]$.

Proof. For statement (1), suppose for every $i, S_{i}=\Lambda_{i}\left[S_{-i}, p\right]$. Consider player $i$ and suppose player $i$ is a strategic complements player. Then $B R_{i}\left(\bar{\mu}_{\vee S_{-i}}\right) \subseteq \Lambda_{i}\left(S_{-i}, p\right)=S_{i}$ implies $\vee B R_{i}\left(\bar{\mu}_{\vee S_{-i}}\right) \leq \vee S_{i}$. Moreover, $\Lambda_{i}\left(S_{-i}, p\right)=S_{i}$ implies that there is $\hat{\mu} \in M^{p}\left[S_{-i}\right]$ such that $\vee S_{i} \in B R_{i}(\hat{\mu})$, and therefore, using strategic complements, $\vee S_{i} \leq \vee B R_{i}\left(\bar{\mu}_{\vee S_{-i}}\right)$. It follows that $\vee S_{i}=\vee B R_{i}\left(\bar{\mu}_{\vee S_{-i}}\right) \in$ $B R_{i}\left(\bar{\mu}_{\vee S_{-i}}\right)=\widetilde{B R}_{i_{H}}\left((\vee S, \wedge S)_{-i_{H}}\right)$. Similarly, it can be shown that $\wedge S_{i}=\wedge B R_{i}\left(\underline{\mu}_{\wedge S_{-i}}\right) \in$ $B R_{i}\left(\underline{\mu}_{\wedge S_{-i}}\right)=\widetilde{B R}_{i_{L}}\left((\vee S, \wedge S)_{-i_{L}}\right)$. A similar argument holds if player $i$ is a strategic substitutes player.

For statement $(2)$, suppose $(x, y)$ is an extremal response equilibrium in $\widetilde{\mathcal{G}}$ with $x \geq y$. For player $i$, consider $a_{i} \in \Lambda_{i}\left(\left[y_{-i}, x_{-i}\right], p\right)$, and let $\mu \in M^{p}\left[y_{-i}, x_{-i}\right]$ be such that $a_{i} \in B R_{i}(\hat{\mu})$. If player $i$ is a strategic complements player, then $B R_{i}\left(\underline{\mu}_{y_{-i}}\right) \sqsubseteq B R_{i}(\mu) \sqsubseteq B R_{i}\left(\bar{\mu}_{x_{-i}}\right)$, and therefore, $y_{i_{L}}=\wedge B R_{i}\left(\underline{\mu}_{y_{-i}}\right) \leq a_{i} \leq \vee B R_{i}\left(\bar{\mu}_{x_{-i}}\right)=x_{i_{H}}$. A similar argument holds if player $i$ is a strategic substitutes player. Identifying $y_{i_{L}}=y_{i}$ and $x_{i_{H}}=x_{i}$ implies that for every player $i$, $\Lambda_{i}\left(\left[y_{-i}, x_{-i}\right], p\right) \subseteq\left[y_{i}, x_{i}\right]$.

For statement $(3)(a)$, only necessity needs to be proved. Suppose $(x, y)$ with $x \geq y$ is an extremal response equilibrium in $\widetilde{\mathcal{G}}$. By statement $(2),[y, x]$ is a $p$-BR set, so that for each player $i$, 
$\Lambda_{i}\left(\left[y_{-i}, x_{-i}\right], p\right) \subseteq\left[y_{i}, x_{i}\right]$. Moreover, by Lemma $2,\left[y_{i}, x_{i}\right] \subseteq \Lambda_{i}\left(\left\{y_{-i}, x_{-i}\right\}, p\right)$. Therefore, for every player $i, \Lambda_{i}\left(\left[y_{-i}, x_{-i}\right], p\right)=\left[y_{i}, x_{i}\right]$, as desired.

For statement $(3)(b)$, suppose $S$ is a $p$-EBR set. By statement $1,(\vee S, \wedge S)$ is an extremal response equilibrium in $\widetilde{\mathcal{G}}$, and therefore, for each player $i$,

$$
S_{i} \subseteq\left[\wedge S_{i}, \vee S_{i}\right] \subseteq \Lambda_{i}\left(\left\{\wedge S_{-i}, \vee S_{i}\right\}, p\right) \subseteq \Lambda_{i}\left(S_{-i}, p\right)=S_{i}
$$

where the second inclusion follows from Lemma 2 and the equality from the assumption that $S$ is a $p$-EBR set. Consequently, for every player $i, S_{i}=\left[\wedge S_{i}, \vee S_{i}\right]$.

Theorem 3 shows the close connection between $p$-EBR sets in $\mathcal{G}$ and extremal response equilibria in $\widetilde{\mathcal{G}}$. Statement (1) shows the necessity of considering extremal response equilibria in order to find $p$-EBR sets in $\mathcal{G}$. When combined with Assumption 1, statement (3)(a) shows the necessity and sufficiency of extremal response equilibria for interval $p$-EBR sets. Statement (3)(b) exhausts all other possibilities by necessitating $p$-EBR sets to be intervals.

Theorem 3 yields straightforward recipes to compute $p$-EBR sets in $\mathcal{G}$. It also provides a natural bijection between $p$-EBR sets in $\mathcal{G}$ and the subset of extremal response equilibria $(x, y)$ in $\widetilde{\mathcal{G}}$ with $x \geq y$. These are formalized as follows.

Corollary 2. Let $\mathcal{G}$ be a monotone game, $\widetilde{\mathcal{G}}$ the auxiliary game at $p \in[0,1]$, and suppose Assumption 1 is satisfied.

1. Every strict Nash equilibrium $(x, y)$ in $\widetilde{\mathcal{G}}$ with $x \geq y$ yields a nonempty $p$-EBR set $[y, x]$ in $\mathcal{G}$.

2. If best responses are singleton-valued, then every Nash equilibrium $(x, y)$ in $\widetilde{\mathcal{G}}$ with $x \geq y$ yields a nonempty $p$-EBR set $[y, x]$ in $\mathcal{G}$.

3. There is a natural bijection between nonempty $p$-EBR sets in $\mathcal{G}$ and extremal response equilibria $(x, y)$ in $\widetilde{\mathcal{G}}$ with $x \geq y$.

Proof. Statement (1) follows immediately from statement (3) of Theorem 3, because a strict Nash equilibrium is an extremal response equilibrium. Statement (2) follows from statement (1), because with singleton best responses, every Nash equilibrium is strict. Statement (3) follows from statement (3) in Theorem 3, using the natural mapping $[y, x] \mapsto(x, y)$ restricted to nonempty $p$-EBR sets in $\mathcal{G}$. 
We now turn our attention to characterizing $p$-MBR sets. The following lemma will be useful.

Lemma 3. Every $p$-BR set contains a $p$-MBR set.

Proof. See Appendix.

Theorem 4. Let $\mathcal{G}$ be a monotone game and $\widetilde{\mathcal{G}}$ the auxiliary game at $p \in[0,1]$.

1. If interval $[y, x]$ is a nonempty $p-M B R$ set in $\mathcal{G}$, then $(x, y)$ is an extremal response equilibrium in $\widetilde{\mathcal{G}}$ with $x \geq y$, and there is no other extremal response equilibrium $\left(x^{\prime}, y^{\prime}\right)$ in $\widetilde{\mathcal{G}}$ with $y \leq$ $y^{\prime} \leq x^{\prime} \leq x$.

2. Suppose Assumption 1 is satisfied as well.

(a) Interval $[y, x]$ is a nonempty $p-M B R$ set in $\mathcal{G}$, if, and only if, $(x, y)$ is an extremal response equilibrium in $\widetilde{\mathcal{G}}$ with $x \geq y$, and there is no other extremal response equilibrium $\left(x^{\prime}, y^{\prime}\right)$ in $\widetilde{\mathcal{G}}$ with $y \leq y^{\prime} \leq x^{\prime} \leq x$.

(b) Every $p-M B R$ set $S$ in $\mathcal{G}$ is of the form $S=[\wedge S, \vee S]$.

Proof. For statement (1), notice that a $p$-MBR set is a $p$-EBR set and therefore, Theorem 3 implies that $(x, y)$ is an extremal response equilibrium in $\widetilde{\mathcal{G}}$ with $x \geq y$. Moreover, if there is a different extremal response equilibrium $\left(x^{\prime}, y^{\prime}\right)$ in $\widetilde{\mathcal{G}}$ with $y \leq y^{\prime} \leq x^{\prime} \leq x$, then Theorem 3 implies that $\left[y^{\prime}, x^{\prime}\right]$ is a strictly smaller $p$-BR set contained in $[y, x]$, a contradiction.

For statement $(2)(\mathrm{a})$, only necessity needs to be proved. Suppose $(x, y)$ is an extremal response equilibrium in $\widetilde{\mathcal{G}}$ with $x \geq y$, and there is no other extremal response equilibrium $\left(x^{\prime}, y^{\prime}\right)$ in $\widetilde{\mathcal{G}}$ with $y \leq y^{\prime} \leq x^{\prime} \leq x$. In this case, Theorem 3 implies that $[y, x]$ is an $p$-BR set in $\mathcal{G}$. Suppose to the contrary that $[y, x]$ is not a $p$-MBR set. Then there is a $p$-BR set $S$ in $\mathcal{G}$ that is a proper subset of this set, that is, $S \subsetneq[y, x]$. By Lemma $3, S$ contains a $p$-MBR set, say, $K \subseteq S$, which, by Proposition 1 , is a $p$-EBR set. If follows from Theorem 3 that $K=[\wedge K, \vee K]$ and also that $(\vee K, \wedge K)$ is an extremal response equilibrium in $\widetilde{\mathcal{G}}$. Finally, $[\wedge K, \vee K] \subseteq S \subsetneq[y, x]$ implies that $(\vee K, \wedge K)$ is a different extremal response equilibrium than $(y, x)$ with $y \leq \wedge K \leq \vee K \leq x$, a contradiction.

Statement (2)(b) follows, because Proposition 1 shows that a $p$-MBR set is a $p$-EBR set, which, by Theorem 3 has the desired form. 
The following examples highlight these results.

Example 2. Consider the following two player, four action game with strategic complements. Each player $i$ has a strategy space $\mathcal{A}_{i}=\{A, B, C, D\}$ with $A \prec B \prec C \prec D$ for $i=1,2$. Payoffs are given in Figure 4 below. This game is a GSC, because each player's payoff has increasing differences. The game has four Nash equilibria, $(A, A),(B, B),(C, C)$, and $(D, D)$.

\section{Player 2}

\begin{tabular}{|c|c|c|c|c|}
\hline \multirow{5}{*}{$\begin{array}{l}\vec{D} \\
\dot{\vec{D}} \\
\dot{\vec{D}}\end{array}$} & $\mathrm{~A}$ & B & $\mathrm{C}$ & $\mathrm{D}$ \\
\hline & (1) & 10,0 & $0,-10$ & $-40,-10$ \\
\hline & 0,10 & 2o & 0,0 & 0,0 \\
\hline & $-10,0$ & 0,0 & 2 & 0,0 \\
\hline & $-10,-40$ & 0,0 & 0,0 & 12 \\
\hline
\end{tabular}

Figure 4: Two player, four action GSC

To fix ideas, suppose we are interested in $p$-dominant equilibria, $p$-MBR sets, and $p$-EBR sets for $p=\frac{3}{4}$. As pointed out in the discussion following Theorem 1, when $\mathcal{G}$ is a GSC, a corresponding auxiliary game $\widetilde{\mathcal{G}}$ can be analyzed in terms of an upper auxiliary game, $\widetilde{\mathcal{G}}_{H}$, and a lower auxiliary game, $\widetilde{\mathcal{G}}_{L}$. Payoffs in the upper auxiliary game at $p=\frac{3}{4}$ are given in Figure 5 . The Nash equilibria in the upper auxiliary game are $(B, B)$ and $(D, D)$. Payoffs in the lower auxiliary game at $p=\frac{3}{4}$ are given in Figure 6. The Nash equilibria in the lower auxiliary game are $(A, A)$ and $(D, D)$. As all Nash equilibria in the auxiliary games are strict, they are also extremal response equilibria.

\section{Player 2}

\begin{tabular}{|c|c|c|c|c|}
\hline \multirow{5}{*}{$\begin{array}{l}-1 \\
\dot{0} \\
\frac{\vec{\sigma}}{2} \\
\end{array}$} & A & B & $\mathrm{C}$ & $\mathrm{D}$ \\
\hline & $-2.5,-2.5$ & $-2.5,0$ & $-10,-7.5$ & $-40,-5$ \\
\hline & $0,-2.5$ & 15 & 0,0 & $0,2.5$ \\
\hline & $-7.5,-10$ & 0,0 & 0,0 & $0,2.5$ \\
\hline & $-5,-40$ & $2.5,0$ & $2.5,0$ & 1212 \\
\hline
\end{tabular}

Figure 5: Upper auxiliary game at $p=\frac{3}{4}$

As $(D, D)$ is the only Nash equilibrium in both upper and lower auxiliary games and it is a strict Nash equilibrium in both games, theorem 2 implies that $(D, D)$ is the unique strict $p$-dominant equilibrium at $p=\frac{3}{4}$. Actions in each of the other three Nash equilibria in the original game are 


\begin{tabular}{|c|c|c|c|c|}
\hline & \multicolumn{4}{|c|}{ Player 2} \\
\hline & $\mathrm{A}$ & B & C & $\mathrm{D}$ \\
\hline \multirow{4}{*}{$\begin{array}{l}\overrightarrow{0} \\
\dot{0} \\
\frac{\overrightarrow{0}}{\tilde{D}_{1}}\end{array}$} & -12 & 10,0 & $2.5,-10$ & $-27.5,-10$ \\
\hline & 0,10 & $7.5,7.5$ & $0,-2.5$ & $0,-2.5$ \\
\hline & $-10,2.5$ & $-2.5,0$ & $-2.5,-2.5$ & $-2.5,-2.5$ \\
\hline & $-10,-27.5$ & $-2.5,0$ & $-2.5,-2.5$ & 5 \\
\hline
\end{tabular}

Figure 6: Lower auxiliary game at $p=\frac{3}{4}$

no longer optimal when each player puts probability $\frac{3}{4}$ or higher on their opponent playing their specified Nash equilibrium action. Corollary 1 implies that $\{D\} \times\{D\}$ is a $p$-MBR set at $p=\frac{3}{4}$.

Theorem 3 tells us how to solve for all $p$-EBR sets. As $(B, B, A, A)$ is an extremal response equilibrium, the interval $[(A, A),(B, B)]=\{A, B\} \times\{A, B\}$ is a $p$-EBR set. Similarly, the interval $[(A, A),(D, D)]$, which is the whole space, and the singleton interval $[(D, D),(D, D)]$ are $p$-EBR sets. There are no other $p$-EBR sets. Theorem 4 shows that the only $p$-MBR sets are the interval $[(A, A),(B, B)]$ and the singleton interval $[(D, D),(D, D)]$.

This is a much easier solution than the conventional approach of considering each possible belief that a player may have about her opponents' actions and for each possible subset of opponent actions.

Example 3. Consider the motivating example from section 2 with payoffs give in Figure 7 . The game has three Nash equilibria: $(A, A),(B, B)$, and $(C, C)$.

Player 2

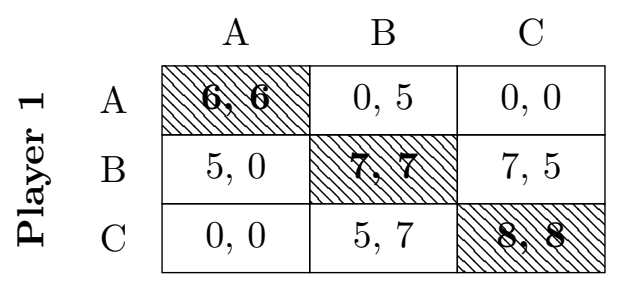

Figure 7: Two player, three action GSC

As this game is a GSC, we can use the upper and lower auxiliary games to find all $p$-dominant equilibria, all $p$-EBR sets, and all $p$-MBR sets for every $p \in[0,1]$. Payoffs for the upper auxiliary game at $p$ are given in Figure 8 and payoffs for the lower auxiliary game at $p$ are given in Figure 9. Nash equilibria in the auxiliary games are listed in Figure 10. 


\section{Player 2}

\begin{tabular}{|c|c|c|c|}
\hline & $\mathrm{A}$ & B & $\mathrm{C}$ \\
\hline \multirow{3}{*}{$\begin{array}{l}-1 \\
\dot{0} \\
\stackrel{\vec{\sigma}}{a} \\
\frac{\vec{a}}{a}\end{array}$} & $6 \mathrm{p}, 6 \mathrm{p}$ & $0,5 p+7(1-p)$ & $0,8(1-p)$ \\
\hline & $5 p+7(1-p), 0$ & 7,7 & $7,5 p+8(1-p)$ \\
\hline & $8(1-p), 0$ & $5 p+8(1-p), 7$ & 8,8 \\
\hline
\end{tabular}

Figure 8: Upper auxiliary game at $p$

\section{Player 2}

\begin{tabular}{|c|c|c|c|}
\hline & $\mathrm{A}$ & $\mathrm{B}$ & $\mathrm{C}$ \\
\hline $\mathrm{A}$ & 6,6 & $6(1-p), 5$ & $6(1-p), 0$ \\
\hline B & $5,6(1-p)$ & $7 \mathrm{p}+5(1-\mathrm{p}), 7 \mathrm{p}+5(1-\mathrm{p})$ & $7 p+5(1-p), 5 p$ \\
\hline $\mathrm{C}$ & $0,6(1-p)$ & $5 p, 7 p+5(1-p)$ & $8 \mathrm{p}, 8 \mathrm{p}$ \\
\hline
\end{tabular}

Figure 9: Lower auxiliary game at $p$

\begin{tabular}{c|c|c}
$p$ & NE: Lower auxiliary game & NE: Upper auxiliary game \\
\hline$p<\frac{1}{8}$ & $(A, A)$ & $(C, C)$ \\
$p \in\left[\frac{1}{8}, \frac{1}{3}\right)$ & $(A, A),(B, B)$ & $(C, C)$ \\
$p \in\left[\frac{1}{3}, \frac{5}{6}\right)$ & $(A, A),(B, B)$ & $(B, B),(C, C)$ \\
$p \in\left[\frac{5}{6}, \frac{7}{8}\right)$ & $(A, A),(B, B),(C, C)$ & $(B, B),(C, C)$ \\
$p \geq \frac{7}{8}$ & $(A, A),(B, B),(C, C)$ & $(A, A),(B, B),(C, C)$
\end{tabular}

Figure 10: Nash equilibria in upper and lower auxiliary games 
Using Theorems 2, 3, and 4, we can read off all $p$-dominant equilibria, all $p$-EBR sets, and all $p$-MBR sets using the list of Nash equilibria for every $p \in[0,1]$. These are listed in Figure 11 . Once again, the exhaustive list of these robustness solutions can be computed easily.

\begin{tabular}{c|c|c|c}
\multicolumn{1}{c|}{$p$} & $p$-EBR sets & $p$-MBR sets & $p$-dominant equilibria \\
\hline$p<\frac{1}{8}$ & $\{A, B, C\} \times\{A, B, C\}$ & $\{A, B, C\} \times\{A, B, C\}$ & None \\
$p \in\left[\frac{1}{8}, \frac{1}{3}\right)$ & $\{A, B, C\} \times\{A, B, C\}$, & $\{B, C\} \times\{B, C\}$ & None \\
& $\{B, C\} \times\{B, C\}$ & & \\
$p \in\left[\frac{1}{3}, \frac{5}{6}\right)$ & $\{A, B, C\} \times\{A, B, C\}$, & $\{B\} \times\{B\}$ & $(B, B)$ \\
& $\{A, B\} \times\{A, B\}$, & & \\
$\{B, C\} \times\{B, C\}$, & & \\
$\left\{\in 5 \frac{5}{6}, \frac{7}{8}\right)$ & $\{A, B, C\} \times\{A, B, C\}$, & $\{B\} \times\{B\}$, & $(B, B)$, \\
& $\{A, B\} \times\{A, B\}$, & $\{C\} \times\{C\}$ & $(C, C)$ \\
& $\{B, C\} \times\{B, C\}$, & & \\
$\{B\} \times \frac{7}{8}$ & $\{B\}$, & & $(A, A)$, \\
& $\{A, B, C\} \times\{A, B, C\}$, & $\{A\} \times\{A\}$, & $(B, B)$, \\
& $\{A, B\} \times\{A, B\}$, & $\{B\} \times\{B\}$, & $(C, C)$ \\
& $\{B, C\} \times\{B, C\}$, & $\{C\} \times\{C\}$ & \\
$\{A\} \times\{A\}$, & & \\
$\{B\} \times\{B\}$, & & & \\
& $\{C\} \times\{C\}$ & &
\end{tabular}

Figure 11: All $p$-EBR sets, $p$-MBR sets and $p$-dominant equilibria

Example 4. Consider a two player game in which player 1 has strategic complements and player 2 has strategic substitutes. Each player has three actions $\{A, B, C\}$ with $A \prec B \prec C$. Payoffs are given in Figure 12. The game has a unique pure strategy Nash equilibrium, $(B, B)$.

\section{Player 2}

\begin{tabular}{cc|c|c|c|} 
& & \multicolumn{1}{c}{$\mathrm{A}$} & \multicolumn{1}{c}{$\mathrm{B}$} & $\mathrm{C}$ \\
\cline { 3 - 5 } \multicolumn{1}{c}{} & $\mathrm{A}$ & 2,0 & 2,1 & 0,2 \\
\cline { 3 - 5 }$\dot{\bar{d}}$ & $\mathrm{~B}$ & 1,2 & & 1,2 \\
\cline { 3 - 5 } $\overrightarrow{\mathrm{C}}$ & $\mathrm{C}$ & 0,2 & 2,1 & 2,0 \\
\cline { 3 - 5 } & & &
\end{tabular}

Figure 12: Game with strategic complements and substitutes

As this game has players with strategic substitutes, there are cross linkages between the payoffs of players in the auxiliary game. For example, payoff for player $1_{H}$ depends on actions of player $2_{H}$, but payoff for player $2_{H}$ depends on actions of player $1_{L}$. Payoff for player $1_{L}$ depends on actions 
of player $2_{L}$, but payoff for player $2_{L}$ depends on actions of player $1_{H}$. These cross linkages imply that the auxiliary game can no longer be decomposed into an upper and lower auxiliary game as in the case of a GSC. The theorems apply without any modifications, but the analysis is carried out in the full auxiliary game.

Payoffs for each player in the auxiliary game at $p$ are summarized in Figure 13.

P2 High

\begin{tabular}{|c|c|c|c|}
\hline & A & B & $\mathrm{C}$ \\
\hline \multirow{2}{*}{ } & $2 p$ & $2 p$ & 0 \\
\hline & 1 & $2 p+1$ & 1 \\
\hline $\mathrm{C}$ & $2-2 p$ & 2 & 2 \\
\hline
\end{tabular}

(a) Payoffs for player $1_{H}$

P2 High

\begin{tabular}{cl|c|c|c|}
\multicolumn{1}{c}{} & \multicolumn{2}{c}{$\mathrm{A}$} & \multicolumn{1}{c}{$\mathrm{B}$} & $\mathrm{C}$ \\
\cline { 3 - 5 } & $\mathrm{A}$ & 0 & 1 & 2 \\
\cline { 3 - 5 } & $\mathrm{B}$ & $2 \mathrm{p}$ & $2 \mathrm{p}+1$ & 2 \\
\cline { 3 - 5 } $\overrightarrow{\mathrm{A}}$ & $\mathrm{C}$ & $2 \mathrm{p}$ & 1 & $2-2 \mathrm{p}$ \\
\cline { 3 - 5 } & & &
\end{tabular}

(c) Payoffs for player $2_{H}$
P2 Low

\begin{tabular}{|c|c|c|c|}
\hline & A & B & $\mathrm{C}$ \\
\hline & 2 & 2 & $2-2 p$ \\
\hline & 1 & $2 \mathrm{p}+1$ & 1 \\
\hline $\mathrm{C}$ & 0 & $2 p$ & $2 \mathrm{p}$ \\
\hline
\end{tabular}

(b) Payoffs for player $1_{L}$

P2 Low

\begin{tabular}{ll|c|c|c|}
\multicolumn{1}{c}{} & \multicolumn{2}{c}{$\mathrm{A}$} & \multicolumn{1}{c}{$\mathrm{B}$} & \multicolumn{1}{c}{$\mathrm{C}$} \\
\cline { 3 - 5 }$\underset{$\cline { 3 - 4 }$}{$\cline { 3 - 4 }$}$ & $\mathrm{A}$ & $2-2 \mathrm{p}$ & 1 & $2 \mathrm{p}$ \\
\cline { 3 - 5 } & $\mathrm{B}$ & 2 & $2 \mathrm{p}+1$ & $2 \mathrm{p}$ \\
\cline { 3 - 5 } & $\mathrm{C}$ & 2 & 1 & 0 \\
\cline { 3 - 5 } & &
\end{tabular}

(d) Payoffs for player $2_{L}$

Figure 13: Payoffs in auxiliary game

For player $1_{H}$, we see that if player $2_{H}$ plays $A$, best response of player $1_{H}$ is $A$, if $p \geq \frac{1}{2}$, and $C$, if $p \leq \frac{1}{2}$. If player $2_{H}$ plays $B$, best response of player $1_{H}$ is $B$, if $p \geq \frac{1}{2}$, and $C$, if $p \leq \frac{1}{2}$. If player $2_{H}$ plays $C$, best response of player $1_{H}$ is $C$ for all $p \in[0,1]$.

For player $1_{L}$, we find that if player $2_{L}$ plays $A$, player $1_{L}$ always best responds with $A$. If player $2_{L}$ plays $B$, best response of player $1_{L}$ is $A$, if $p \leq \frac{1}{2}$, and $B$, if $p \geq \frac{1}{2}$. If player $2_{L}$ plays $C$, best response of player $1_{L}$ is $A$, if $p \leq \frac{1}{2}$, and $C$, if $p \geq \frac{1}{2}$.

For player $2_{H}$, one can easily verify that if player $1_{L}$ plays $A$, player $2_{H}$ always best responds with $C$. If player $1_{L}$ plays $B$, best response of player $2_{H}$ is $C$, if $p \leq \frac{1}{2}$, and $B$, if $p \geq \frac{1}{2}$. If player $1_{L}$ plays $C$, best response of player $2_{H}$ is $C$, if $p \leq \frac{1}{2}$, and $A$, if $p \geq \frac{1}{2}$.

Lastly, for player $2_{L}$, if player $1_{H}$ plays $A$, best response of player $2_{L}$ is $A$, if $p \leq \frac{1}{2}$, and $C$, if $p \geq \frac{1}{2}$. If player $1_{H}$ plays $B$, best response of player $2_{L}$ is $A$, if $p \leq \frac{1}{2}$, and $B$, if $p \geq \frac{1}{2}$. If player $1_{H}$ 
plays $C$, player $2_{L}$ always best responds with $A$.

Solving for Nash equilibria in the auxiliary game, it is easy to check that for $p<\frac{1}{2}$, the unique Nash equilibrium in the auxiliary game is $\left(x_{1_{H}}, x_{2_{H}}, y_{1_{L}}, y_{2_{L}}\right)=(C, C, A, A)$, and for $p \geq$ $\frac{1}{2}$, there are two Nash equilibria in the auxiliary game: $\left(x_{1_{H}}, x_{2_{H}}, y_{1_{L}}, y_{2_{L}}\right)=(B, B, B, B)$ and $\left(x_{1_{H}}, x_{2_{H}}, y_{1_{L}}, y_{2_{L}}\right)=(C, C, A, A)$.

Using Theorems 2, 3, and 4, we conclude that for $p<\frac{1}{2}$, the entire action space $\{A, B, C\} \times$ $\{A, B, C\}$ is the unique $p$-EBR set and the unique $p$-MBR set, and there is no $p$-dominant equilibrium. For $p \geq \frac{1}{2}$, there are two $p$-EBR sets, one given by the entire space $\{A, B, C\} \times\{A, B, C\}$ and the other is the singleton $\{B\} \times\{B\}$, there is a unique $p$-MBR set $\{B\} \times\{B\}$, and a unique p-dominant equilibrium $(B, B)$.

The payoffs of this game can also be represented in one combined payoff matrix, which is illustrated in Figure 14. The top (bottom) row in each cell contains the payoffs of Player 1's (Player 2's) high player on the left and Player 1's (Player 2's) low player on the right. The payoffs for the Nash equilibria for each case are in boldface. 


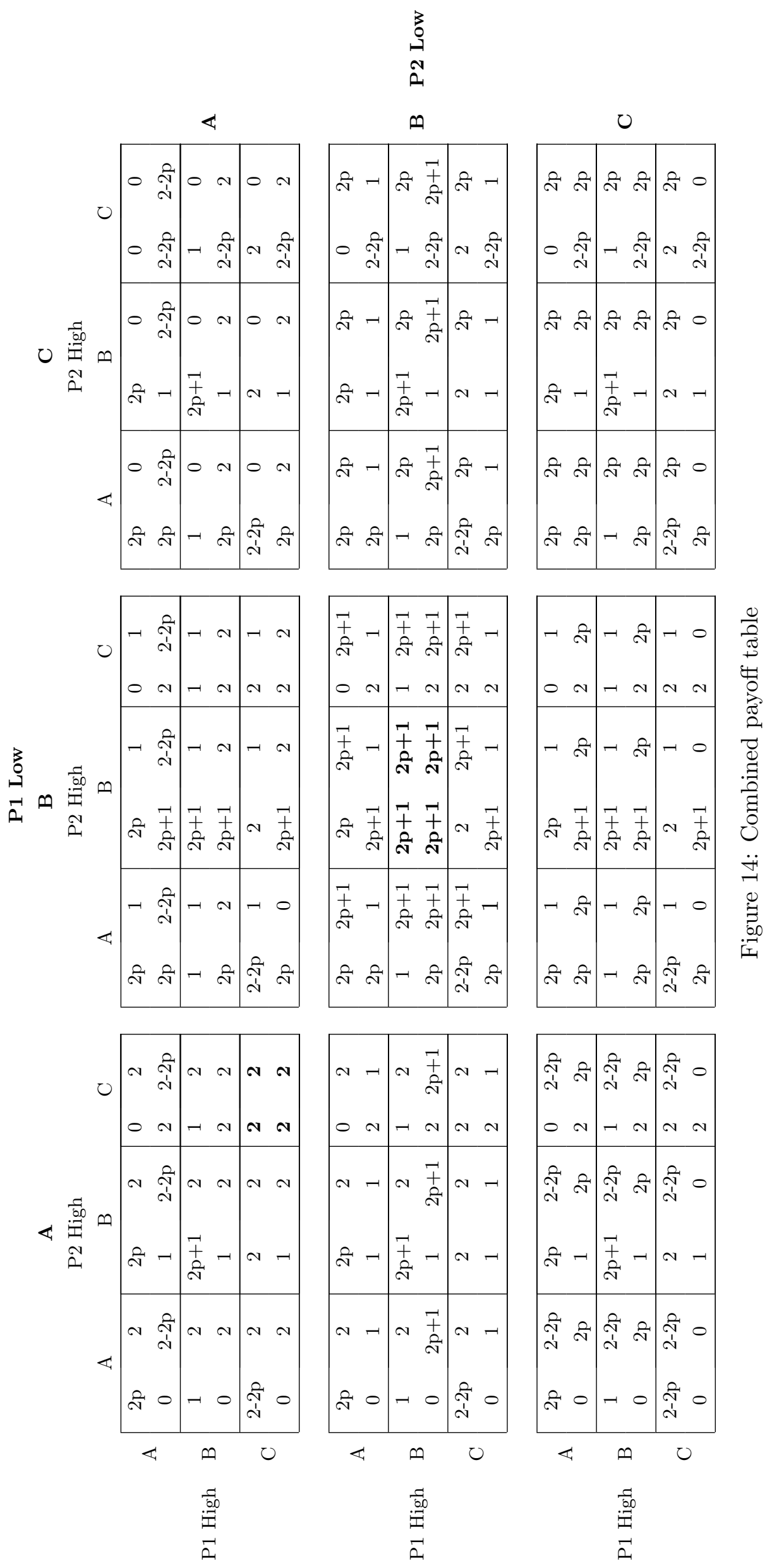


The next example is a Cournot duopoly with continuous action spaces.

Example 5. Consider a Cournot duopoly in which each firm $i \in\{1,2\}$ chooses quantity of output $q_{i} \in[0,50]$ and has a constant marginal cost of production $M C\left(q_{i}\right)=10$. Suppose inverse market demand is given by $P\left(q_{1}, q_{2}\right)=100-q_{1}-q_{2}$. Profit of each firm $i$ can be written as $\pi_{i}\left(q_{i}, q_{-i}\right)=$ $\left(90-q_{i}-q_{-i}\right) q_{i}$. This is a GSS and profit functions are concave in own action. Therefore, Assumption 1 is satisfied.

Let $p \in[0,1]$ be arbitrary. As both firms have strategic substitutes, payoffs in the auxiliary game are given by, for each $i \in\{1,2\}$ and each $(x, y) \in \mathcal{A}_{H} \times \mathcal{A}_{L}$,

$$
\begin{aligned}
& \tilde{\pi}_{i_{H}}(x, y)=\pi_{i}\left(x_{i}, p \delta_{y_{-i}}+(1-p) \delta_{0}\right)=\left(90-x_{i}-p y_{-i}\right) x_{i}, \text { and } \\
& \tilde{\pi}_{i_{L}}(x, y)=\pi_{i}\left(y_{i}, p \delta_{x_{-i}}+(1-p) \delta_{50}\right)=\left(40+50 p-y_{i}-p x_{-i}\right) y_{i} .
\end{aligned}
$$

The auxiliary game has a unique Nash equilibrium given by

$$
\left(q_{1_{H}}^{*}, q_{2_{H}}^{*}, q_{1_{L}}^{*}, q_{2_{L}}^{*}\right)=\left(\frac{180-40 p-50 p^{2}}{4-p^{2}}, \frac{180-40 p-50 p^{2}}{4-p^{2}}, \frac{80+10 p}{4-p^{2}}, \frac{80+10 p}{4-p^{2}}\right) .
$$

Theorems 2, 3, and 4 imply that the interval $\left[\left(q_{1_{L}}^{*}, q_{2_{L}}^{*}\right),\left(q_{1_{H}}^{*}, q_{2_{H}}^{*}\right)\right] \subseteq \mathcal{A}$ is the unique $p$-EBR set and unique $p$-MBR set in the original game, and therefore, for $p<1$ there is no $p$-dominant equilibrium. For $p=1$, the unique $p$-EBR set, unique $p$-MBR set, and unique $p$-dominant equilibrium are given by the unique Nash equilibrium $\left(q_{1}^{*}, q_{2}^{*}\right)=(30,30)$.

Notice that for $p=0$, this interval contains those actions that survive the first round of the process of iteratively deleting strictly dominated actions, and as $p$ increases to 1 , this interval shrinks toward the unique Nash equilibrium in the original game, $\left(q_{1}^{*}, q_{2}^{*}\right)=(30,30)$. Figure 15 illustrates the boundaries of these intervals for $p=0, p=0.5$ and $p=1$.

To the best of the authors' knowledge, there is no other solved example with continuous action spaces in the literature. Solving for $p$-EBR sets, $p$-MBR sets and $p$-dominant equilibria in this case requires keeping track of best response sets over a continuum of beliefs, each over a continuum of actions, and over subsets of continuum of actions. Our results continue to apply to such cases, simply by solving for pure strategy Nash equilibria in the auxiliary game using standard optimization techniques. 


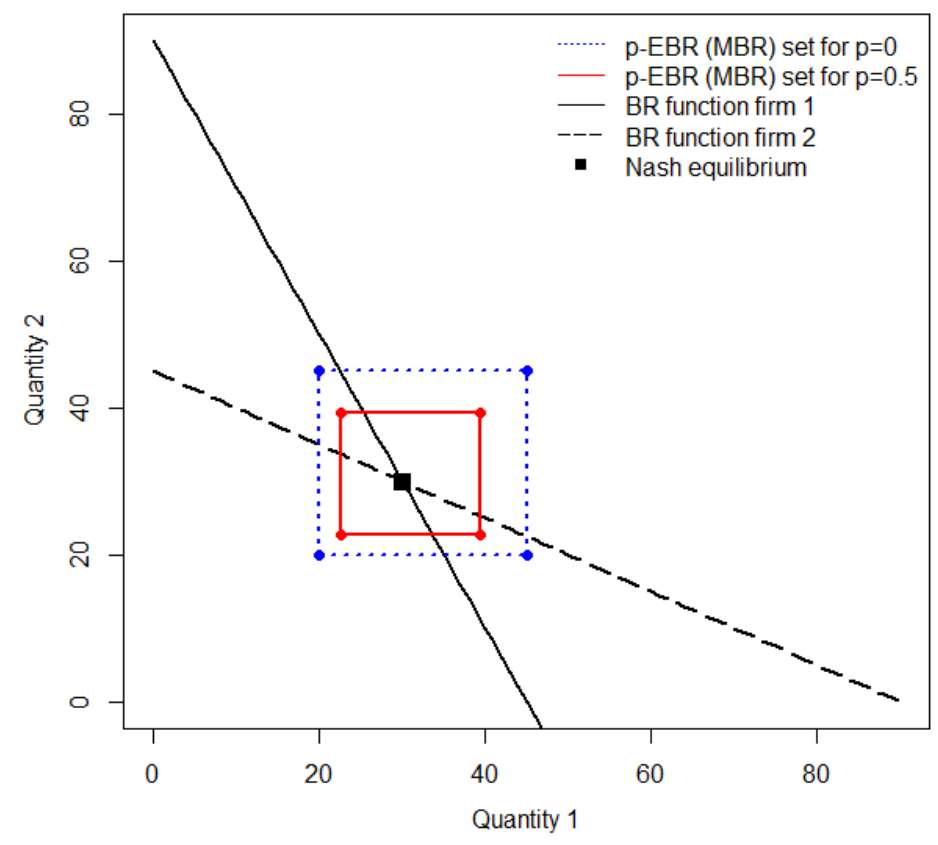

Figure 15: p-EBR (MBR) sets and Nash equilibrium in Cournot duopoly

\section{Structure of class of $p$-EBR sets and $p$-MBR sets}

Let $\mathcal{S}_{E}$ be the collection of nonempty $p$-EBR sets in $\mathcal{G}$, and $\mathcal{S}_{M}$ be the collection of nonempty $p$-MBR sets in $\mathcal{G}$. From Theorems 3 and 4 , we know that every nonempty $p$-EBR set and every nonempty $p$-MBR set is a nonempty interval in $\mathcal{A}$. As the set of nonempty intervals is partially ordered by the lattice set order, it follows that both $\mathcal{S}_{E}$ and $\mathcal{S}_{M}$ are partially ordered in the lattice set order.

In order to understand the structure of $\mathcal{S}_{E}$ and $\mathcal{S}_{M}$, it is helpful to have a notion of strict strategic complements and strict strategic substitutes, as follows. For subsets $A, B$ of $\mathbb{R}^{n}, A$ is completely lower than $B$, denoted $A \sqsubset_{c} B$, if $\forall a \in A, \forall b \in B, a<b$. (As usual, $a<b$ means $a \leq b$ and $a \neq b$.)

Definition 3. Let $\mathcal{G}$ be a monotone game and $\widetilde{\mathcal{G}}$ be the auxiliary game at $p \in[0,1]$.

Player $i \in \mathcal{I}$ has strict strategic complements, if for its high copy $i_{H}$, for every $x, x^{\prime} \in \mathcal{A}_{H}$ and for every $y \in \mathcal{A}_{L}, x_{-i_{H}}<x_{-i_{H}}^{\prime} \Rightarrow \widetilde{B R}_{i_{H}}\left((x, y)_{-i_{H}}\right) \sqsubset_{c} \widetilde{B R}_{i_{H}}\left(\left(x^{\prime}, y\right)_{-i_{H}}\right)$, and for its low copy $i_{L}$, for every $y, y^{\prime} \in \mathcal{A}_{L}$ and for every $x \in \mathcal{A}_{H}, y_{-i_{L}}<y_{-i_{L}}^{\prime} \Rightarrow \widetilde{B R}_{i_{L}}\left((x, y)_{-i_{L}}\right) \sqsubset_{c} \widetilde{B R}_{i_{L}}\left(\left(x, y^{\prime}\right)_{-i_{L}}\right)$. 
Player $i \in \mathcal{I}$ has strict strategic substitutes, if for its high copy $i_{H}$, for every $y, y^{\prime} \in \mathcal{A}_{L}$ and for every $x \in \mathcal{A}_{H}, y_{-i_{L}}<y_{-i_{L}}^{\prime} \Rightarrow \widetilde{B R}_{i_{H}}\left(\left(x, y^{\prime}\right)_{-i_{H}}\right) \sqsubset_{c} \widetilde{B R}_{i_{H}}\left((x, y)_{-i_{H}}\right)$, and for its low copy $i_{L}$, for every $x, x^{\prime} \in \mathcal{A}_{H}$ and for every $y \in \mathcal{A}_{L}, x_{-i_{H}}<x_{-i_{H}}^{\prime} \Rightarrow \widetilde{B R}_{i_{L}}\left(\left(x^{\prime}, y\right)_{-i_{L}}\right) \sqsubset_{c} \widetilde{B R}_{i_{L}}\left((x, y)_{-i_{L}}\right)$.

The next theorem shows that under Assumption 1 , when $\mathcal{G}$ is a game with strategic complements, both $\mathcal{S}_{E}$ and $\mathcal{S}_{M}$ are a complete lattice. On the other hand, if $\mathcal{G}$ is a monotone game in which there are only two players with strict strategic substitutes or in which there is one player with strict strategic substitutes and one player with strict strategic complements, then both $\mathcal{S}_{E}$ and $\mathcal{S}_{M}$ are totally unordered. In other words, moving away from a GSC even in some minimal sense completely destroys the complete lattice structure of $p$-EBR sets and $p$-MBR sets. This is consistent with results for the set of Nash equilibria in these types of games, as shown in Roy and Sabarwal (2008) and in Monaco and Sabarwal (2016).

Theorem 5. Let $\mathcal{G}$ be a monotone game, $\widetilde{\mathcal{G}}$ be the auxiliary game at $p \in[0,1]$, and suppose Assumption 1 holds.

1. If $\mathcal{G}$ is a $G S C$, then both $\mathcal{S}_{E}$ and $\mathcal{S}_{M}$ are complete lattices.

2. If $\mathcal{G}$ has either (1) two players with strict strategic substitutes, or (2) one player with strict strategic substitutes and one player with strict strategic complements, then both $\mathcal{S}_{E}$ and $\mathcal{S}_{M}$ are totally unordered.

Proof. For statement (1), we show first that $\mathcal{S}_{E}$ is a complete lattice. Consider a collection of non-empty $p$-EBR sets indexed by $t$, denoted $\left\{\left[y^{t}, x^{t}\right] \in \mathcal{S}_{E} \mid t \in T\right\}$. Then for every $t \in T$, $\left(x^{t}, y^{t}\right)$ is an extremal response equilibrium in $\widetilde{\mathcal{G}}$ with $x^{t} \geq y^{t}$. Thus, for every $t$, for every $i_{H}$, $x_{i_{H}}^{t} \in \vee \widetilde{B R}_{i_{H}}\left((x, y)_{-i_{H}}\right)$, and for every $i_{L}, y_{i_{L}}^{t} \in \wedge \widetilde{B R}_{i_{L}}\left((x, y)_{-i_{L}}\right)$.

As each player is a strategic complements player, payoff of a high type is affected only by payoffs of other high types and payoff of a low type is affected only by payoffs of other low types. That is, $\vee \widetilde{B R}_{i_{H}}\left((x, y)_{-i_{H}}\right)$ does not depend on $y$ and $\wedge \widetilde{B R}_{i_{L}}\left((x, y)_{-i_{L}}\right)$ does not depend on $x$. Denote this as $\vee \widetilde{B R}_{i_{H}}\left(x_{-i_{H}}\right)$ and $\wedge \widetilde{B R}_{i_{L}}\left(y_{-i_{L}}\right)$ and notice that strategic complements implies that the corresponding joint best response functions denoted by $\vee \widetilde{B R}(x)$ and $\wedge \widetilde{B R}(y)$ are increasing, and therefore, the set of fixed points of each is a complete lattice. 
As each $x^{t}$ is a fixed point of $\vee \widetilde{B R}$ and each $y^{t}$ is a fixed point of $\wedge \widetilde{B R}$, let $\underline{x}$ be the infimum of $\left\{x^{t}\right\}$ over the set of fixed points of $\vee \widetilde{B R}$, and $\bar{x}$ be the supremum, and similarly, let $\underline{y}$ be the infimum of $\left\{y^{t}\right\}$ over the set of fixed points of $\wedge \widetilde{B R}$ and $\bar{y}$ be the supremum. Then $\underline{x} \leq \wedge\left\{x^{t}\right\}$, $\bar{x} \geq \vee\left\{x^{t}\right\}, \underline{y} \leq \wedge\left\{y^{t}\right\}$, and $\bar{y} \geq \vee\left\{y^{t}\right\}$, and moreover, $(\underline{x}, \underline{y})$ and $(\bar{x}, \bar{y})$ are both extremal response equilibria in $\widetilde{\mathcal{G}}$ with $\underline{x} \geq \underline{y}$ and $\bar{x} \geq \bar{y}$. Consequently, both $[\underline{y}, \underline{x}]$ and $[\bar{y}, \bar{x}]$ are nonempty $p$-EBR sets in $\mathcal{G}$.

We show that $[\underline{y}, \underline{x}]=\inf _{\mathcal{S}_{E}}\left\{\left[y^{t}, x^{t}\right] \in \mathcal{S}_{E} \mid t \in T\right\}$ and $[\bar{y}, \bar{x}]=\sup _{\mathcal{S}_{E}}\left\{\left[y^{t}, x^{t}\right] \in \mathcal{S}_{E} \mid t \in T\right\}$. As for every $t, \underline{y} \leq y^{t} \leq \bar{y}$ and $\underline{x} \leq x^{t} \leq \bar{x}$, it follows that for every $t$,

$$
[\underline{y}, \underline{x}] \sqsubseteq\left[y^{t}, x^{t}\right] \sqsubseteq[\bar{y}, \bar{x}]
$$

Therefore, $[\underline{y}, \underline{x}]$ is a lower bound for $\left\{\left[y^{t}, x^{t}\right] \in \mathcal{S}_{E} \mid t \in T\right\}$ and $[\bar{y}, \bar{x}]$ an upper bound. To check that $[\underline{y}, \underline{x}]$ is the infimum, consider an abritrary nonempty $p$-EBR set $[\hat{y}, \hat{x}]$ that is also a lower bound. Then $(\hat{x}, \hat{y})$ is an extremal response equilibrium in $\widetilde{\mathcal{G}}$ with $\hat{x} \geq \hat{y}, \hat{x}$ is an extremal response equilibrium in the upper auxiliary game $\widetilde{\mathcal{G}}_{H}, \hat{y}$ is an extremal response equilibrium in the lower auxiliary game $\widetilde{\mathcal{G}}_{L}$, and for every $t, \hat{y} \leq y^{t}$ and $\hat{x} \leq x^{t}$. As $\underline{x}$ is the largest of the equilibria smaller than $x^{t}$, it follows that $\hat{x} \leq \underline{x}$ and similarly, $\hat{y} \leq \underline{y}$. That is, $[\hat{y}, \hat{x}] \sqsubseteq[\underline{y}, \underline{x}]$, and therefore, $[\underline{y}, \underline{x}]$ is the infimum. Similarly, $[\bar{y}, \bar{x}]$ is the supremum. This shows that $\mathcal{S}_{E}$ is a complete lattice.

To show that $\mathcal{S}_{M}$ is a complete lattice, consider a collection of nonempty $p$-MBR sets indexed by $t$, denoted $\left\{\left[y^{t}, x^{t}\right] \in \mathcal{S}_{M} \mid t \in T\right\}$. As $\mathcal{S}_{M} \subseteq \mathcal{S}_{E}$, each $\left[y^{t}, x^{t}\right]$ is a nonempty $p$-EBR set. For this collection of sets, let $\underline{x}, \bar{x}, \underline{y}$, and $\bar{y}$ be defined as above. Then $[\underline{y}, \underline{x}]$ is a nonempty $p$-EBR set and as $\mathcal{S}_{E}$ is complete, $[\underline{y}, \underline{x}]=\inf _{\mathcal{S}_{E}}\left\{\left[y^{t}, x^{t}\right] \in \mathcal{S}_{M} \mid t \in T\right\}$. We show that $[\underline{y}, \underline{x}]$ contains a unique $p$-MBR set of the form $[\underline{y}, \hat{x}]$ for some $\hat{x} \preceq \underline{x}$ and this is the desired infimum. A similar argument produces the desired supremum.

Notice first that every nonempty $p$-EBR set that is a subset of $[\underline{y}, \underline{x}]$ must be of the form $\left[\underline{y}, x^{\prime}\right]$ for some $x^{\prime} \leq \underline{x}$. Consider a nonempty $p$-EBR set $[y, x] \subset[\underline{y}, \underline{x}]$. As $[\underline{y}, \underline{x}]$ is an infimum, it cannot be that $\underline{y}<y$, because if $\underline{y}<y$, then there is $t^{\prime}$ such that $\underline{y} \leq y^{t^{\prime}}<y \leq x \leq \underline{x} \leq x^{t^{\prime}}$, and this contradicts the fact that $\left[y^{t^{\prime}}, x^{t^{\prime}}\right]$ is a $p$-MBR set.

Notice next that as $[\underline{y}, \underline{x}]$ is a $p$-BR set, Lemma 3 implies that it contains at least one $p$-MBR 
set, (which must also be a $p$-EBR set,) and therefore, by the previous argument is of the form $\left[y, x^{\prime}\right]$ for some $x^{\prime} \leq \underline{x}$. Moreover, by Proposition 1 , every $p$-MBR set is disjoint from every other $p$-MBR set, and therefore, there can be at most one $p$-MBR set that is a subset of $[\underline{y}, \underline{x}]$. It follows that there is a unique $\hat{x} \leq \underline{x}$ such that $[\underline{y}, \hat{x}]$ is the only $p$-MBR set contained in $[\underline{y}, \underline{x}]$. It is immediate that $[\underline{y}, \hat{x}] \sqsubseteq[y, \underline{x}]$, and therefore, $[\underline{y}, \hat{x}]$ is a lower bound for $\left\{\left[y^{t}, x^{t}\right] \in \mathcal{S}_{M} \mid t \in T\right\}$. To check that this is the infimum, consider a $p$-MBR set $\left[y^{\prime}, x^{\prime}\right]$ that is a larger lower bound, that is,

$$
[\underline{y}, \hat{x}] \sqsubseteq\left[y^{\prime}, x^{\prime}\right] \sqsubseteq[\underline{y}, \underline{x}]
$$

This implies that $\underline{y}=y^{\prime}$ and $\hat{x} \leq x^{\prime} \leq \underline{x}$. As there is only one such $p$-MBR set, it follows that $\hat{x}=x^{\prime}$, and therefore, $[\underline{y}, \hat{x}]$ is the infimum. A similar argument shows that there is unique $\hat{y} \geq \underline{y}$ such that $[\hat{y}, \bar{x}]$ is a $p$-MBR set and $[\hat{y}, \bar{x}]=\sup _{\mathcal{S}_{M}}\left\{\left[y^{t}, x^{t}\right] \in \mathcal{S}_{M} \mid t \in T\right\}$. Together, this shows that $\mathcal{S}_{M}$ is a complete lattice.

For statement (2), suppose $\mathcal{G}$ has two players with strict strategic substitutes, say, players 1 and 2, without loss of generality. Consider two distinct nonempty $p$-EBR sets $[y, x]$ and $\left[y^{\prime}, x^{\prime}\right]$, and suppose $[y, x] \sqsubseteq\left[y^{\prime}, x^{\prime}\right]$. Then $x \leq x^{\prime}, y \leq y^{\prime}$, and at least one inequality is strict. Suppose $x<x^{\prime}$. As case 1 , suppose $x_{-1}<x_{-1}^{\prime}$. In this case, $x_{-1_{H}}<x_{-1_{H}}^{\prime}$ and strict strategic substitutes implies $\widetilde{B R}_{1_{L}}\left(\left(x^{\prime}, y\right)_{-1_{L}}\right) \sqsubset_{c} \widetilde{B R}_{1_{L}}\left((x, y)_{-1_{L}}\right)$. Therefore, $\wedge \widetilde{B R}_{1_{L}}\left(\left(x^{\prime}, y\right)_{-1_{L}}\right)<\wedge \widetilde{B R}_{1_{L}}\left((x, y)_{-1_{L}}\right)$. As $(x, y)$ and $\left(x^{\prime}, y^{\prime}\right)$ are extremal response equilibria, $y_{1_{L}}^{\prime}=\wedge \widetilde{B R}_{1_{L}}\left(\left(x^{\prime}, y^{\prime}\right)_{-1_{L}}\right)=\wedge \widetilde{B R}_{1_{L}}\left(\left(x^{\prime}, y\right)_{-1_{L}}\right)$ and $y_{1_{L}}=\wedge \widetilde{B R}_{1_{L}}\left((x, y)_{-1_{L}}\right)$, and this implies $y_{1_{L}}^{\prime}<y_{1_{L}}$, a contradiction to $y \leq y^{\prime}$. As case 2 , suppose $x_{-1}=x_{-1}^{\prime}$ and $x_{1}<x_{1}^{\prime}$. Then $x_{-2_{H}}<x_{-2_{H}}^{\prime}$, and the same argument as in the previous case shows that $y_{2_{L}}^{\prime}<y_{2_{L}}$, a contradiction to $y \leq y^{\prime}$. A similar argument applies to the case $y<y^{\prime}$. Therefore, $\mathcal{S}_{E}$ is totally unordered. As $\mathcal{S}_{M}$ is a subset of $\mathcal{S}_{E}$, it follows that $\mathcal{S}_{M}$ is totally unordered as well.

Now suppose $\mathcal{G}$ has one player with strict strategic substitutes (say, player 1) and one player with strict strategic complements (say, player 2). Consider two distinct nonempty $p$-EBR sets $[y, x]$ and $\left[y^{\prime}, x^{\prime}\right]$, and suppose $[y, x] \sqsubseteq\left[y^{\prime}, x^{\prime}\right]$. Then $x \leq x^{\prime}, y \leq y^{\prime}$, and at least one inequality is strict. Suppose $x<x^{\prime}$. If $x_{-1}<x_{-1}^{\prime}$, the same argument as in the case above yields a contradiction. If $x_{-1}=x_{-1}^{\prime}$ and $x_{1}<x_{1}^{\prime}$, then $x_{-2}<x_{-2}^{\prime}$, and therefore, $x_{-2_{H}}<x_{-2_{H}}^{\prime}$. Player 
2 has strict strategic complements implies $\widetilde{B R}_{2_{H}}\left((x, y)_{-2_{H}}\right) \sqsubset_{c} \widetilde{B R}_{2_{H}}\left(\left(x^{\prime}, y\right)_{-2_{H}}\right)$, and therefore, $\vee \widetilde{B R}_{2_{H}}\left((x, y)_{-2_{H}}\right)<\vee \widetilde{B R}_{2_{H}}\left(\left(x^{\prime}, y\right)_{-2_{H}}\right)$. As $(x, y)$ and $\left(x^{\prime}, y^{\prime}\right)$ are extremal response equilibria, $x_{2_{H}}=\vee \widetilde{B R}_{2_{H}}\left((x, y)_{-2_{H}}\right)$ and $x_{2_{H}}^{\prime}=\vee \widetilde{B R}_{2_{H}}\left(\left(x^{\prime}, y^{\prime}\right)_{-2_{H}}\right)=\vee \widetilde{B R}_{2_{H}}\left(\left(x^{\prime}, y\right)_{-2_{H}}\right)$, and this implies $x_{2_{H}}<x_{2_{H}}^{\prime}$, a contradiction to $x_{-1}=x_{-1}^{\prime}$. A similar argument applies to the case $y<y^{\prime}$. Therefore, $\mathcal{S}_{E}$ is totally unordered. As $\mathcal{S}_{M}$ is a subset of $\mathcal{S}_{E}$, it follows that $\mathcal{S}_{M}$ is totally unordered as well.

Example 6. Consider the GSC in Example 3 and consider the case when $p \geq \frac{7}{8}$. In this case, as shown in Figure 11, the game has six $p$-EBR sets, $\{A, B, C\} \times\{A, B, C\},\{A, B\} \times\{A, B\}$, $\{B, C\} \times\{B, C\},\{A\} \times\{A\},\{B\} \times\{B\}$, and $\{C\} \times\{C\}$. The last three sets are also the three $p$-MBR sets for $p \geq \frac{7}{8}$. These sets are illustrated in Figure 16. By Theorem 5, the collection of $p$-EBR sets and the collection of $p$-MBR are complete lattices. In other words, for any two $p$-EBR (or $p$-MBR) sets, their infimum and supremum (in the lattice set order) are $p$-EBR (or $p$-MBR) sets as well. For example, consider the $p$-EBR sets $\{A, B, C\} \times\{A, B, C\}$ and $\{B\} \times\{B\}$. The infimum of these two sets is $\{A, B\} \times\{A, B\}$, the supremum is $\{B, C\} \times\{B, C\}$, and both are $p$-EBR sets.

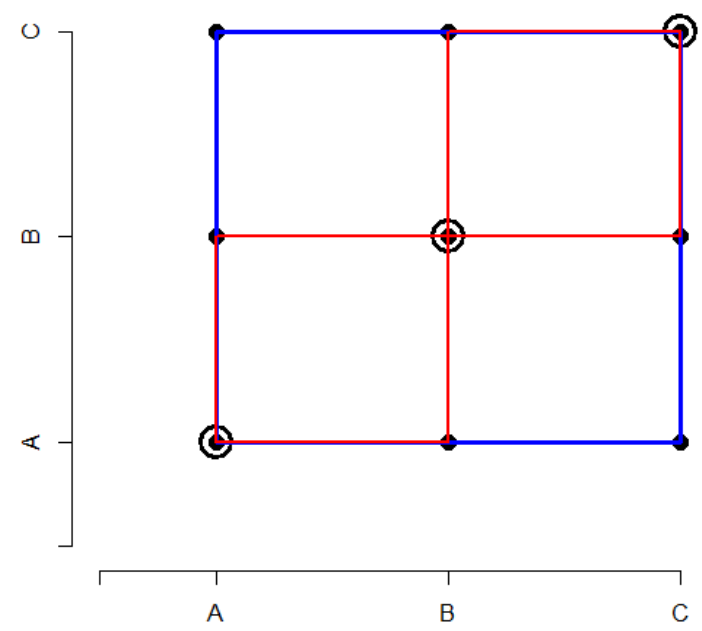

structure 2.png

Figure 16: $p$-EBR and $p$-MBR sets for $p \geq \frac{7}{8}$

Example 7. Consider the monotone game with one strategic substitutes and one strategic complements player from Example 4. Recall that for $p \geq \frac{1}{2}$, this game has two $p$-EBR sets, $\{A, B, C\} \times$ $\{A, B, C\}$ as well as $\{B\} \times\{B\}$. These sets are illustrated in Figure 17. By Theorem 5, the collection of $p$-EBR sets is totally unordered. In particular, $\{B\} \times\{B\} \nsubseteq\{A, B, C\} \times\{A, B, C\}$, because 
$(B, B) \wedge(A, A) \notin\{B\} \times\{B\}$, and $\{A, B, C\} \times\{A, B, C\} \nsubseteq\{B\} \times\{B\}$, because $(C, C) \vee(B, B) \notin$ $\{B\} \times\{B\}$.

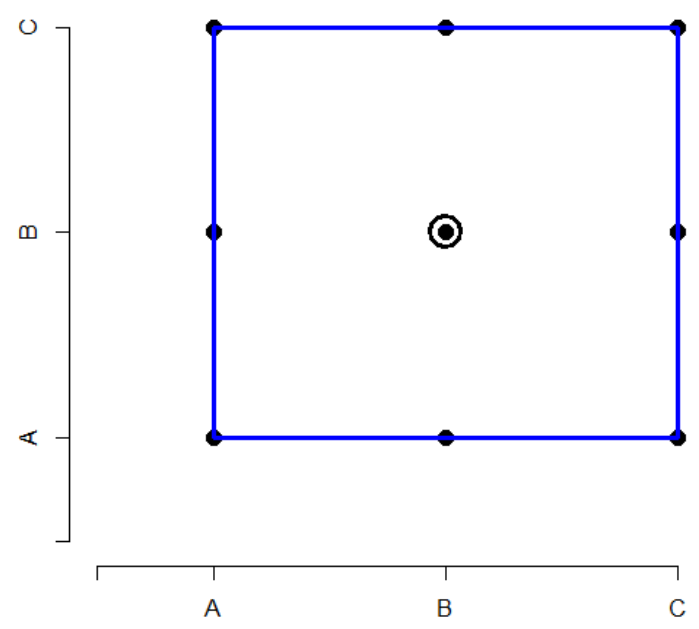

Figure 17: $p$-EBR and $p$-MBR sets for $p \geq \frac{1}{2}$

\section{Conclusion}

We show that for monotone games, solving for $p$-dominant equilibrium and $p$-MBR set is equivalent to finding a corresponding Nash equilibrium in an auxiliary game. This shows that traditional methods for finding equilibria may be applied to the complete information auxiliary game, which allows us to solve for robust solution concepts in a straightforward, systematic manner and avoiding the need to consider uncountably many beliefs for each player. Moreover, as use of Nash equilibrium is ubiquitous, our results make robust solution concepts more accessible to a broader audience, facilitating their broader use. Furthermore, by establishing a bijection between robust solutions in the original game and corresponding Nash equilibria in the auxiliary game, we are able to establish new results on characteristics of entire classes of robust solutions.

For $p$-dominant equilibrium, we show that a profile of actions $a^{*}$ is a $p$-dominant equilibrium in a monotone game, if, and only, if $\left(a^{*}, a^{*}\right)$ is a Nash equilibrium in the auxiliary game, and $a^{*}$ is a strict $p$-dominant equilibrium in a monotone game, if, and only if, $\left(a^{*}, a^{*}\right)$ is a strict Nash equilibrium in the auxiliary game. This gives a bijection between $p$-dominant equilibria in the 
original game and particular types of Nash equilibria in the auxiliary game.

For $p$-EBR set, we show that in a monotone game, under Assumption 1, an interval $[y, x]$ is a $p$-EBR set, if, and only if, $(x, y)$ with $x \geq y$ is an extremal response equilibrium in the auxiliary game. This gives a bijection between $p$-EBR sets in the original game and particular types of Nash equilibria in the auxiliary game. An appropriate specialization yields a characterization of all $p$-MBR sets and a bijection to a subclass of the Nash equilibria in the auxiliary game.

These characterizations show that we may find $p$-dominant equilibrium, $p$-EBR set, and $p$-MBR set in a monotone game by considering particular pure strategy Nash equilibria in the auxiliary game. Several examples highlight applications of these results.

We also provide structure theorems for the class of $p$-EBR sets and for the class of $p$-MBR sets in monotone games. In games with strategic complements, each class is a complete lattice. With minimal extensions beyond that, each class is totally unordered. 


\section{References}

Carlsson, H., and E. van Damme (1993): "Global games and equilibrium selection," Econometrica, 61(5), 989-1018.

Choi, M., And L. Smith (2017): “Ordinal aggregation results via Karlin's variation diminishing property," Journal of Economic Theory, 168, 1-11.

Christensen, F. (2017): "A necessary and sufficient condition for a unique maximum with an application to potential games," Economics Letters, 161, 120-123.

Durieu, J., P. Solal, And O. Tercieux (2011): "Adaptive learning and p-best response sets," International Journal of Game Theory, 40(4), 735-747.

Frankel, D. M., S. Morris, and A. Pauzner (2003): "Equilibrium selection in global games with strategic complementarities," Journal of Economic Theory, 108(1), 1-44.

Harsanyi, J. C., And R. Selten (1988): A general theory of equilibrium selection in games. MIT Press.

Hoffmann, E. J., And T. SABARwal (2019): "Global games with strategic complements and substitutes," Games and Economic Behavior, 118, 72-93.

KAJII, A., AND S. MorRIS (1997): "The robustness of equilibria to incomplete information," Econometrica, 65(6), 1283-1309.

Monaco, A. J., And T. Sabarwal (2016): "Games with strategic complements and substitutes," Economic Theory, 62(1-2), 65-91.

Morris, S., R. Rob, And H. S. Shin (1995): "p-Dominance and belief potential," Econometrica, pp. $145-157$.

Morris, S., AND T. Ui (2005): "Generalized potentials and robust sets of equilibria," Journal of Economic Theory, 124(1), 45-78.

Mutoh, I. (2006): "Mathematical economics in Vienna between the Wars," in Advances in mathematical economics, ed. by S. Kusuoka, and T. Maruyama, vol. 8, pp. 167-195. Springer. 
Roy, S., and T. Sabarwal (2008): "On the (Non-)Lattice Structure of the Equilibrium Set in Games with Strategic Substitutes," Economic Theory, 37(1), 161-169.

Tercieux, O. (2006a): "p-Best response set," Journal of Economic Theory, 131(1), 45-70. (2006b): "p-Best response set and the robustness of equilibria to incomplete information," Games and Economic Behavior, 56(2), 371-384.

Topkis, D. M. (1978): "Minimizing a submodular function on a lattice," Operations research, 26(2), 305-321.

Veinoti, A. F. (1989): "Lattice programming," Unpublished notes from lectures delivered at Johns Hopkins University.

Zhou, L. (1994): "The set of Nash equilibria of a supermodular game is a complete lattice," Games and Economic Behavior, 7(2), 295-300. 


\section{Appendix}

\section{Proof of Proposition 2}

Proof. Let $i \in \mathcal{I}, a_{-i}, a_{-i}^{\prime} \in \mathcal{A}_{-i}$ be such that $a_{-i}^{\prime} \geq a_{-i}$, and consider $a_{i}^{\prime \prime}, a_{i}^{\prime}, a_{i} \in \mathcal{A}_{i}$ such that $a_{i}^{\prime \prime}>a_{i}^{\prime}>a_{i}$, and let $p \in[0,1]$. Notice that

$$
\begin{gathered}
\pi_{i}\left(a_{i}^{\prime}, p \delta_{a_{-i}}+(1-p) \delta_{a_{-i}^{\prime}}\right)-\pi_{i}\left(a_{i}, p \delta_{a_{-i}}+(1-p) \delta_{a_{-i}^{\prime}}\right)= \\
p\left[\pi_{i}\left(a_{i}^{\prime}, a_{-i}\right)-\pi_{i}\left(a_{i}, a_{-i}\right)\right]+(1-p)\left[\pi_{i}\left(a_{i}^{\prime}, a_{-i}\right)-\pi_{i}\left(a_{i}, a_{-i}^{\prime}\right)\right] \geq \\
p\left[\pi_{i}\left(a_{i}^{\prime \prime}, a_{-i}\right)-\pi_{i}\left(a_{i}^{\prime}, a_{-i}\right)\right]+(1-p)\left[\pi_{i}\left(a_{i}^{\prime \prime}, a_{-i}\right)-\pi_{i}\left(a_{i}^{\prime}, a_{-i}^{\prime}\right)\right]= \\
\pi_{i}\left(a_{i}^{\prime \prime}, p \delta_{a_{-i}}+(1-p) \delta_{a_{-i}^{\prime}}\right)-\pi_{i}\left(a_{i}^{\prime}, p \delta_{a_{-i}}+(1-p) \delta_{a_{-i}^{\prime}}\right),
\end{gathered}
$$

so that $\pi_{i}$ satisfies decreasing returns in $a_{i}$ against each belief $p \delta_{a_{-i}}+(1-p) \delta_{a_{-i}^{\prime}}$. Suppose that $x, y \in B R_{i}\left(p \delta_{a_{-i}}+(1-p) \delta_{a_{-i}^{\prime}}\right)$ are such that $x>y$, and suppose $z \in \mathcal{A}_{i}$ is such that $x \geq z \geq y$. If $z \notin B R_{i}\left(p \delta_{a_{-i}}+(1-p) \delta_{a_{-i}^{\prime}}\right)$, then by decreasing returns in $a_{i}$,

$$
\begin{gathered}
0>\pi_{i}\left(z, p \delta_{a_{-i}}+(1-p) \delta_{a_{-i}^{\prime}}\right)-\pi_{i}\left(y, p \delta_{a_{-i}}+(1-p) \delta_{a_{-i}^{\prime}}\right) \geq \\
\pi_{i}\left(x, p \delta_{a_{-i}}+(1-p) \delta_{a_{-i}^{\prime}}\right)-\pi_{i}\left(z, p \delta_{a_{-i}}+(1-p) \delta_{a_{-i}^{\prime}}\right),
\end{gathered}
$$

contradicting the optimality of $x$. Hence $B R_{i}\left(p \delta_{a_{-i}}+(1-p) \delta_{a_{-i}^{\prime}}\right)$ is interval-valued.

\section{Proof of Lemma 2}

Proof. Notice first that for each $a_{-i}, a_{-i}^{\prime} \in \mathcal{A}_{-i}$, the best response correspondence $B R_{i}\left(p \delta_{a_{-i}}+\right.$ $\left.(1-p) \delta_{a_{-i}^{\prime}}\right)$ is upper hemicontinuous in $p$. This follows from Berge's theorem, because the function $\left(a_{i}, p\right) \mapsto p \pi_{i}\left(a_{i}, a_{-i}\right)+(1-p) \pi_{i}\left(a_{i}, a_{-i}^{\prime}\right)$ is continuous, and therefore,

$$
B R_{i}\left(p \delta_{a_{-i}}+(1-p) \delta_{a_{-i}^{\prime}}\right)=\underset{a_{i} \in \mathcal{A}_{i}}{\operatorname{argmax}}\left(p \pi_{i}\left(a_{i}, a_{-i}\right)+(1-p) \pi_{i}\left(a_{i}, a_{-i}^{\prime}\right)\right)
$$

is upper hemicontinuous in $p$. 
Suppose without loss of generality that player $i$ is a strategic complements player, so that $\pi_{i}$ satisfies increasing differences in $\left(a_{i}, a_{-i}\right)$. The case when $\pi_{i}$ satisfies decreasing differences in $\left(a_{i}, a_{-i}\right)$ can be proven similarly.

We now show that $\left[y_{i}, x_{i}\right] \subseteq \Lambda_{i}\left(\left\{y_{-i}, x_{-i}\right\}, p\right)$. The remainder of the proof follows along the lines of the intermediate value theorem given for correspondences in Mutoh (2006), but adapted for our purposes. Because player $i \in \mathcal{I}$ is a complements player and $(x, y)$ is an extremal response equilibrium, the definition of an auxiliary game implies

$$
x_{i}=\vee \widetilde{B R}_{i_{H}}\left((x, y)_{-i}\right)=\vee B R_{i}\left(\bar{\mu}_{x_{-i}}\right) \text { and } y_{i}=\wedge \widetilde{B R}_{i_{L}}\left((x, y)_{-i}\right)=\wedge B R_{i}\left(\underline{\mu}_{y_{-i}}\right)
$$

so that $y_{i}, x_{i} \in \Lambda_{i}\left(\left\{y_{-i}, x_{-i}\right\}, p\right)$. Also, because $B R_{i}\left(y_{-i}\right)$ and $B R_{i}\left(x_{-i}\right)$ are best responses to beliefs $\delta_{y_{-i}}$ and $\delta_{x_{-i}}$, respectively, both of which put probability 1 on $\left\{y_{-i}, x_{-i}\right\}$, we have that $B R_{i}\left(y_{-i}\right), B R_{i}\left(x_{-i}\right) \in \Lambda_{i}\left(\left\{y_{-i}, x_{-i}\right\}, p\right)$ as well. Using arguments similar to those in the proof to Theorem 1 , it is readily verified that $T=\left\{\underline{\mu}_{y_{-i}}, \delta_{y_{-i}}\right\}, T^{\prime}=\left\{\delta_{y_{-i}}, \delta_{x_{-i}}\right\}$, and $T^{\prime \prime}=\left\{\delta_{x_{-i}}, \bar{\mu}_{x_{-i}}\right\}$ are partially ordered sets. By strategic complementarities, when $\geq_{F}$ is a partial order, $\mu \geq_{F} \nu$ implies $B R_{i}(\nu) \sqsubseteq B R_{i}(\mu)$, and hence we can write $\left[y_{i}, x_{i}\right]$ as

$$
\left[y_{i}, x_{i}\right]=\left[y_{i}, \wedge B R_{i}\left(y_{-i}\right)\right] \cup\left[\wedge B R_{i}\left(y_{-i}\right), \vee B R_{i}\left(x_{-i}\right)\right] \cup\left[\vee B R_{i}\left(x_{-i}\right), x_{i}\right]
$$

where the endpoints of all three intervals above are included in $\Lambda_{i}\left(\left\{y_{-i}, x_{-i}\right\}, p\right)$. Thus, the result follows by showing that all three intervals are included in $\Lambda_{i}\left(\left\{y_{-i}, x_{-i}\right\}, p\right)$.

We first show that $\left[y_{i}, \wedge B R_{i}\left(y_{-i}\right)\right]$ is included in $\Lambda_{i}\left(\left\{y_{-i}, x_{-i}\right\}, p\right)$. Consider the beliefs $\mu_{\alpha} \in$ $\Delta\left(A_{-i}\right)$ given by

$$
\mu_{\alpha}=[\alpha p+(1-\alpha)] \delta_{y_{-i}}+[\alpha(1-p)] \delta_{\wedge \mathcal{A}_{-i}}
$$

for each $\alpha \in[0,1]$. Note that for each such $\alpha, \mu_{\alpha}$ puts at least probability $p$ on $y_{-i}$, and hence on $\left\{y_{-i}, x_{-i}\right\}$. Also, by the discussion above, $\wedge B R_{i}\left(y_{-i}\right) \in B R_{i}\left(\mu_{0}\right)$, and $y_{i} \in B R_{i}\left(\mu_{1}\right)$. Suppose there exists some $z \in \mathcal{A}_{i}$ such that $y_{i}<z<\wedge B R_{i}\left(y_{-i}\right)$, but $z \notin \Lambda_{i}\left(\left\{y_{-i}, x_{-i}\right\}, p\right)$. Then $y_{i}<z<x_{i}$. Consider the sets

$$
K_{z}^{-}=\left\{\alpha \in[0,1] \mid \forall x \in B R_{i}\left(\mu_{\alpha}\right), x<z\right\},
$$


and

$$
K_{z}^{+}=\left\{\alpha \in[0,1] \mid \forall x \in B R_{i}\left(\mu_{\alpha}\right), z<x\right\}
$$

First, notice that $K_{z}^{-}$and $K_{z}^{+}$are non-empty: From above, we know $y_{i} \in B R_{i}\left(\mu_{1}\right)$ and $y_{i}<z$. If some other $x \in B R_{i}\left(\mu_{1}\right)$ were such that $x \geq z$, then because $B R_{i}\left(\mu_{1}\right)$ is interval-valued, we would have $z \in B R_{i}\left(\mu_{1}\right)$ as well. Because the belief $\mu_{1}$ puts probability at least $p$ on $\left\{y_{-i}, x_{-i}\right\}$, we would have $z \in \Lambda_{i}\left(\left\{y_{-i}, x_{-i}\right\}, p\right)$, a contradiction. Hence, $1 \in K_{z}^{-}$. Likewise, $0 \in K_{z}^{+}$. Next, notice that $[0,1]=K_{z}^{-} \cup K_{z}^{+}$: Since each $\mu_{\alpha}$ puts at least probability $p$ on $\left\{y_{-i}, x_{-i}\right\}$, then for each $\alpha \in[0,1]$, $B R_{i}\left(\mu_{\alpha}\right) \subseteq \Lambda_{i}\left(\left\{y_{-i}, x_{-i}\right\}, p\right)$. Suppose $\alpha \in[0,1]$ is such that for some $x \in B R_{i}\left(\mu_{\alpha}\right), x>z$. If there exists some other $y \in B R_{i}\left(\mu_{\alpha}\right)$ such that $z>y$, then because $B R_{i}\left(\mu_{\alpha}\right)$ is interval-valued, we have that $z \in B R_{i}\left(\mu_{\alpha}\right) \subseteq \Lambda_{i}\left(\left\{y_{-i}, x_{-i}\right\}, p\right)$, a contradiction. Hence, for each $\alpha$ such that for some $x \in B R_{i}\left(\mu_{\alpha}\right)$ we have $x>z$, it follows that $\alpha \in K_{z}^{+}$. Likewise, for each $\alpha$ such that for some $x \in B R_{i}\left(\mu_{\alpha}\right)$ we have $z>x$, it follows that $\alpha \in K_{z}^{-}$. Because each $\alpha \in[0,1]$ satisfies one of the two requirements, it follows that $[0,1] \subseteq K_{z}^{-} \cup K_{z}^{+}$, establishing equality.

Lastly, upper hemicontinuity of $B R_{i}\left(\mu_{\alpha}\right)$ in $\alpha$ implies that $K_{z}^{-}$is an open set, because it is the upper inverse of the open set $(-\infty, z)$ and $K_{z}^{+}$is an open set, because it is the upper inverse of the open set $(z, \infty)$. Taken together, this contradicts the connectedness of $[0,1]$. Therefore, $\left[y_{i}, \wedge B R_{i}\left(y_{-i}\right)\right] \subseteq \Lambda_{i}\left(\left\{y_{-i}, x_{-i}\right\}, p\right)$.

A similar argument shows that $\left[\vee B R_{i}\left(x_{-i}\right), x_{i}\right] \subseteq \Lambda_{i}\left(\left\{y_{-i}, x_{-i}\right\}, p\right)$ by considering beliefs of the form

$$
\mu_{\alpha}=[\alpha p+(1-\alpha)] \delta_{x_{-i}}+[\alpha(1-p)] \delta_{\vee A_{-i}},
$$

and likewise it follows that $\left[\wedge B R_{i}\left(y_{-i}\right), \vee B R_{i}\left(x_{-i}\right)\right] \subseteq \Lambda_{i}\left(\left\{y_{-i}, x_{-i}\right\}, p\right)$ by considering beliefs of the form

$$
\mu_{\alpha}=(1-\alpha) \delta_{y_{-i}}+\alpha \delta_{x_{-i}}
$$




\section{Proof of Lemma 3}

Proof. This follows from the same reasoning as part (1) of Theorem 1 in Tercieux (2006a). Suppose that $Z \subseteq \mathcal{A}$ is a $p-\mathrm{BR}$ set, and consider

$$
Q=\{S \subseteq Z \mid S \in P, \Lambda[S, p] \subseteq S\}
$$

the collection of $p$-BR subsets of $Z$, which are partially ordered by set inclusion $\subseteq$. Notice that $Q$ is non-empty, as $Z$ is by assumption a $p-\mathrm{BR}$ set, and $Z \subseteq Z$. By the Hausdorff maximal principle, there exists a chain $Q^{\prime} \subseteq Q$ such that $Q^{\prime}$ is not a strict sub-family of any other chain $T \subseteq Q$. For each player $i \in \mathcal{I}$, define

$$
\hat{S}_{i}=\bigcap_{S^{\prime} \in Q^{\prime}} S_{i}^{\prime}
$$

which, as the intersection of closed (compact) and nonempty sets, is itself closed and nonempty. Furthermore, since $\hat{S}_{i} \subseteq Z_{i}$, and $Z_{i}$ is compact, $\hat{S}_{i}$ is compact as well. Hence, $\hat{S}=\prod_{i \in \mathcal{I}} \hat{S}_{i} \in P$.

Since $\hat{S} \subseteq S^{\prime}$ for each $S^{\prime} \in Q^{\prime}$, it follows that $\Lambda_{i}\left[\hat{S}_{-i}, p\right] \subseteq \Lambda_{i}\left[S_{-i}^{\prime}, p\right] \subseteq S_{i}^{\prime}$ for each $i \in \mathcal{I}$ and all $S^{\prime} \in Q^{\prime}$, where the last inequality follows because each $S^{\prime}$ is a $p-\mathrm{BR}$ set. Hence,

$$
\Lambda_{i}\left[\hat{S}_{-i}, p\right] \subseteq \bigcap_{S^{\prime} \in Q^{\prime}} S_{i}^{\prime}=\hat{S}_{i}
$$

and hence $\hat{S}$ is a $p-\mathrm{BR}$ set. Note that $\hat{S}$ is a minimal $p-\mathrm{BR}$ set, for if there exists some strict subset $M \subset \hat{S}$ which is a $p-\mathrm{BR}$ set, then $Q^{\prime}$ is a strict sub-family of the chain $\{M\} \cup Q^{\prime}$, contradicting the maximality of $Q^{\prime}$. 